\title{
The Engineering Merit of the "Effective Period" of Bilinear Isolation Systems
}

\author{
Nicos Makris ${ }^{* 1 a}$ and Georgios Kampas ${ }^{2 b}$ \\ ${ }^{1}$ :Division of Structures, Department of Civil Engineering, University of Patras, Greece \\ ${ }^{2}:$ Robertou Galli 27, Athens 11742, Greece
}

\begin{abstract}
This paper examines whether the "effective period" of bilinear isolation systems, as defined invariably in most current design codes, expresses in reality the period of vibration that appears in the horizontal axis of the design response spectrum. Starting with the free vibration response, the study proceeds with a comprehensive parametric analysis of the forced vibration response of a wide collection of bilinear isolation systems subjected to pulse and seismic excitations. The study employs Fourier and Wavelet analysis together with a powerful time domain identification method for linear systems known as the Prediction Error Method. When the response history of the bilinear system exhibits a coherent oscillatory trace with a narrow frequency band as in the case of free vibration or forced vibration response from most pulselike excitations, the paper shows that the "effective period" $=T_{\text {eff }}$ of the bilinear isolation system is a dependable estimate of its vibration period; nevertheless, the period associated with the second slope of the bilinear system $=T_{2}$ is an even better approximation regardless the value of the dimensionless strength, $Q /\left(K_{2} u_{y}\right)=1 / \alpha-1$, of the system. As the frequency content of the excitation widens and the intensity of the acceleration response history fluctuates more randomly, the paper reveals that the computed vibration period of the systems exhibits appreciably scattering from the computed mean value. This suggests that for several earthquake excitations the mild nonlinearities of the bilinear isolation system dominate the response and the expectation of the design codes to identify a "linear" vibration period has a marginal engineering merit.
\end{abstract}

Keywords: Seismic Isolation; Equivalent Linearization; Bilinear Behavior; System Identification; Health Monitoring; Earthquake Protection.

\section{Introduction}

Starting in the late 1950s researchers began recognizing the importance of studying the response of structures deforming into their inelastic range and this led to the development of the inelastic response spectrum. In parallel with the development of inelastic response spectra in the 1960s (Veletsos and Newmark 1960, Veletsos et al. 1969, Veletsos and Vann 1971), there has been significant effort in developing equivalent linearization techniques (Caughey 1960;1963, Roberts and Spanos 2003, Crandall 2006) in order to define equivalent linear parameters (natural periods and damping ratios) of equivalent linear systems that exhibit comparable response values to those of the nonlinear systems (Iwan and Gates 1979, Iwan 1980).

In the mid 1970s seismic base isolation has emerged as a practical and economical alternative to conventional structural design (Kelly et al. 1977, Kelly 1986, Buckle and Mayes 1990). Given that the two most practical and widely accepted type of isolation bearings are the lead rubber bearing and the spherical sliding bearing which both exhibit a bilinear behavior, the bilinear hysteretic system is by now the most widely used model in describing the nonlinear behavior of practical seismic isolated systems. Despite that the behavior of the most practical seismic isolation systems is bilinear, the fundamental concept of seismic isolation, as expressed in most current design codes (AASHTO 1991, NZMWD 1983, FEMA 1998, Eurocode 2009 among others), is that an isolation system shall offer a flexible support so that

a:Corresponding Author. Professor, Email: nmakris@upatras.gr

${ }^{\mathrm{b}}$ :Civil Engineer, former PhD student in University of Patras, Email: gkampas@upatras.gr 
the period of vibration is lengthen sufficiently to reduce the force response. Accordingly, while the behavior of the most practical isolation systems is bilinear, all design codes invariably ask the design engineer to work with a vibration period -that is the "isolation" period. In view of this demand the concept of equivalent linear parameters has become central in the analysis and design of seismic isolated structures and this led to the wide acceptance of the "effective period" and the associated "effective stiffness". The main scope of this work is to access the engineering merit of these quantities.

This work has been mainly motivated from system identification studies on seismic isolated structures in which modal periods and damping ratios are expected to be extracted from recorded response histories above and below isolators. This work shows that there are appreciable differences between the first modal period extracted with identification techniques and the "effective period" which according to the design codes is expected to be the "period of vibration". During the effort to uncover these differences this work also concludes that when the response history of the bilinear system has a coherent oscillatory trace with a narrow frequency band, the effective period, $T_{\text {eff }}$ as derived from the nonexisting effective stiffness, $K_{\text {eff }}$, of the bilinear system (in which iterations are needed to be determined) is a dependable estimate of its vibration period; nevertheless, the period associated with the second slope of the bilinear system $=T_{2}$ is an even better approximation of the vibration period regardless the value of the dimensionless strength, $Q /\left(K_{2} u_{y}\right)=1 / \alpha-1$. Accordingly, whenever the concept of a vibration period is meaningful, the effective period, $T_{e f f}$ can be replaced with $T_{2}$ which is known a priori.

Initially, the "effective stiffness" $=K_{\text {eff }}$, was introduced by practicing engineers in an effort to reach an estimate for the peak forces that develop in seismic isolated structures with bilinear (inelastic) behavior by simply employing a statically equivalent linear analysis. At present, $K_{\text {eff }}$, together with the corresponding effective period, $T_{\text {eff }}$ and the associated effective damping coefficient, $\xi_{\text {eff }}$, consist the most widely used quantities for estimating through an iterative procedure peak inelastic displacements and the associated peak shear forces/overturning moments according to most current design codes (AASHTO 1991, FEMA 1998, Eurocode 2009 among others).

The main challenges that $T_{\text {eff }}$ is facing are: (a) that it depends on the unknown peak inelastic displacement; and therefore, iterations on the design spectrum are required to reach convergence, (b) in seismic isolation applications it has not been established to what extent the effective period, $T_{\text {eff }}=2 \pi \sqrt{m / K_{\text {eff }}}$, (what appears in the horizontal axis of the design spectrum) is indeed the vibration period (the time needed to complete one cycle) of a mass, $m$, supported on a system with bilinear behavior (mass isolated on lead rubber or spherical sliding bearings); and (c) that in several occasions there are significant departures of the peak inelastic deformation/force of the bilinear system from the elastic displacement/force of the "effective" linear system.

The concerns with challenge (b) have been expressed indirectly in several review-type publications and textbooks (Naeim 2001, Naeim and Kelly 1999) where, while they introduce, $T_{\text {eff }}=2 \pi \sqrt{m / K_{\text {eff }}}$ as the "vibration period" of a structure isolated on bearings with bilinear behavior ( $T_{I}=T_{\text {eff }}$-what appears in the horizontal axis of the spectrum); for the case of spherical sliding bearings (where the first slope is 200 to 500 times larger than the second slope - a much more aggressive bilinear behavior), the concept of the effective period, $T_{\text {eff }}=2 \pi \sqrt{m / K_{\text {eff }}}$ is suddenly abandoned, and the isolation period (vibration period), is derived from the second slope of the system, $T_{I}=T_{2}=2 \pi \sqrt{R / g}$. Fig. 1 (left) plots typical bilinear force displacement loops that correspond to a lead rubber bearing (say strength 
$Q=0.05 \mathrm{mg}$, period that corresponds to the second slope, $K_{2}$, is $2.5 \mathrm{sec}$, and $K_{1} / K_{2}=1 / \alpha=10$ ); while Fig. 1 (right) plots the corresponding force displacement loops from a spherical sliding bearing $\left(T_{I}=2.5 s \rightarrow R=1.55 m, u_{y}=0.00025 m\right.$ and coefficient of friction $f=0.05$; therefore $K_{1} / K_{2}=1 / \alpha=1+f R / u_{y}=311$ ).

The above inconsistency, where the entire concept of the effective period is abandoned in the very case where the bilinear behavior is most pronounced (large difference between $K_{1}$ and $K_{2}$ ) remains confusing to the non-expert and most importantly uncovers potential technical weaknesses in the concept of the "effective period", $T_{\text {eff }}$. This paper revisits the practical significance and engineering merit of the "effective period", $T_{\text {eff }}$, while investigates to what extent it expresses the oscillatory characteristics of an isolated structure. In this regard, the dynamic response of several bilinear hysteretic systems (with different normalized strengths and second slopes) is investigated for three types of excitation: free vibration, pulse-type forced vibration and earthquake forced vibration. The investigation methods include: similitude, Fourier spectrum response analysis, wavelet response analysis, and the Prediction Error Method response analysis.
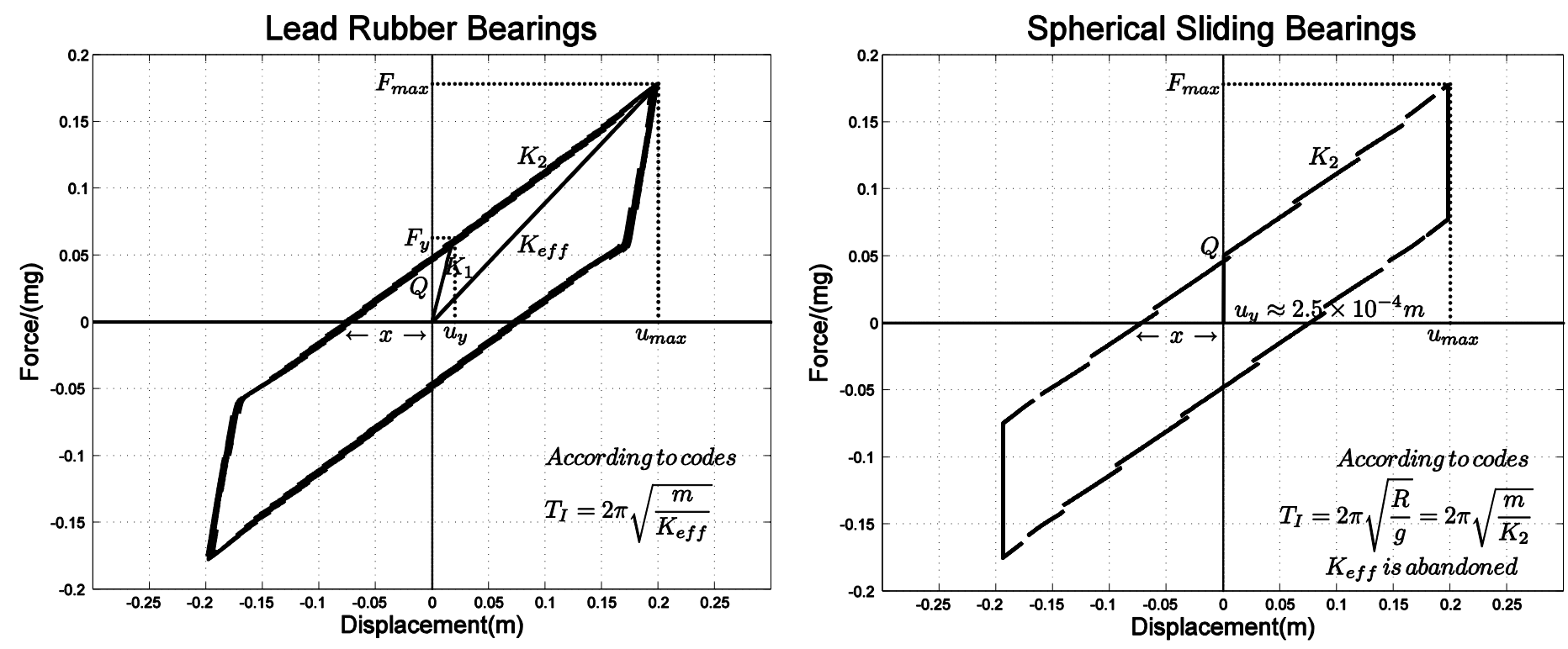

Figure 1. The hysteretic loops of lead rubber bearings (left, say $\left.u_{y}=2 \mathrm{~cm}=20 \mathrm{~mm}\right)$ and spherical-sliding bearings (right, say $u_{y}=0.25 \mathrm{~mm}$ ) together with the inconsistent code definition of the isolation period, $T_{I}$, as the yield displacement decreases ( $K_{\text {eff }}$ is abandoned in the right plot).

\section{Review of Design Codes and Related Past Publications}

The currently available design specifications AASHTO (1991), FEMA (1998), IBC (2000), Eurocode (2009) among others use invariably the equivalent linear static procedure. Details on the specific steps followed by the most widely accepted codes can be found in Mayes et al. (1991), Hwang and Sheng (1993); (1994) as well as and in the original documents of the abovementioned design specifications. Below we only revisit the main steps followed by the 1991 AASHTO Guide Specification for Seismic Isolation Design given that all subsequent design specifications follow a similar approach. 


\subsection{The AASHTO Guide specifications}

The code specifications that established the quantity, $K_{\text {eff }}$, of the bilinear system shown in Fig. 1 as a key quantity for the response analysis of seismically isolated structures is apparently the 1991 AASHTO Guide Specifications for Seismic Isolation Design. At that time the code did not make a distinction between the Design Base Earthquake (DBE) and the Maximum Credible Earthquake (MCE) and merely offers the design spectrum presented in Fig. 2. According to the AASHTO Guide specification the statically equivalent seismic force is given by

$$
F=\sum K_{e f f} d_{i}
$$

where $\sum K_{\text {eff }}$ is the sum of the effective linear stiffnesses of all bearings supporting the superstructure and $d_{i}$ is the displacement across the isolation bearings given by

$$
d_{i}=S_{D}=\frac{10 A S_{i} T_{e f f}}{B}=\frac{T_{e f f}^{2}}{4 \pi^{2}} S_{A},
$$

where $A, S_{i}$ and $B$ are the acceleration, site and damping coefficients offered in the AASHTO Guide specifications and the effective period, $T_{\text {eff }}$ is given by

$$
T_{\text {eff }}=2 \pi \sqrt{\frac{W}{\sum K_{e f f} g}} .
$$

The effective linear stiffness $K_{\text {eff }}$ of the isolators used in the analysis shall be calculated at the design displacement, however iterations on the design spectrum are needed given that the effective period $T_{\text {eff }}$ as offered by equation (3) updates the maximum displacement as defined by equation (2).

The conceptual weakness of the Statically Equivalent Seismic Force Procedure offered by equation (1) - (3) is that while its ultimate goal is to reach an estimate for the peak design "static" forces, the estimation of the isolators displacement $d_{i}=S_{D}$ involves the effective period, $T_{\text {eff }}$ given by equation (3). By involving the effective period, $T_{\text {eff }}$, the "static" procedure also takes a stand on the oscillatory character of the bilinear system; and the

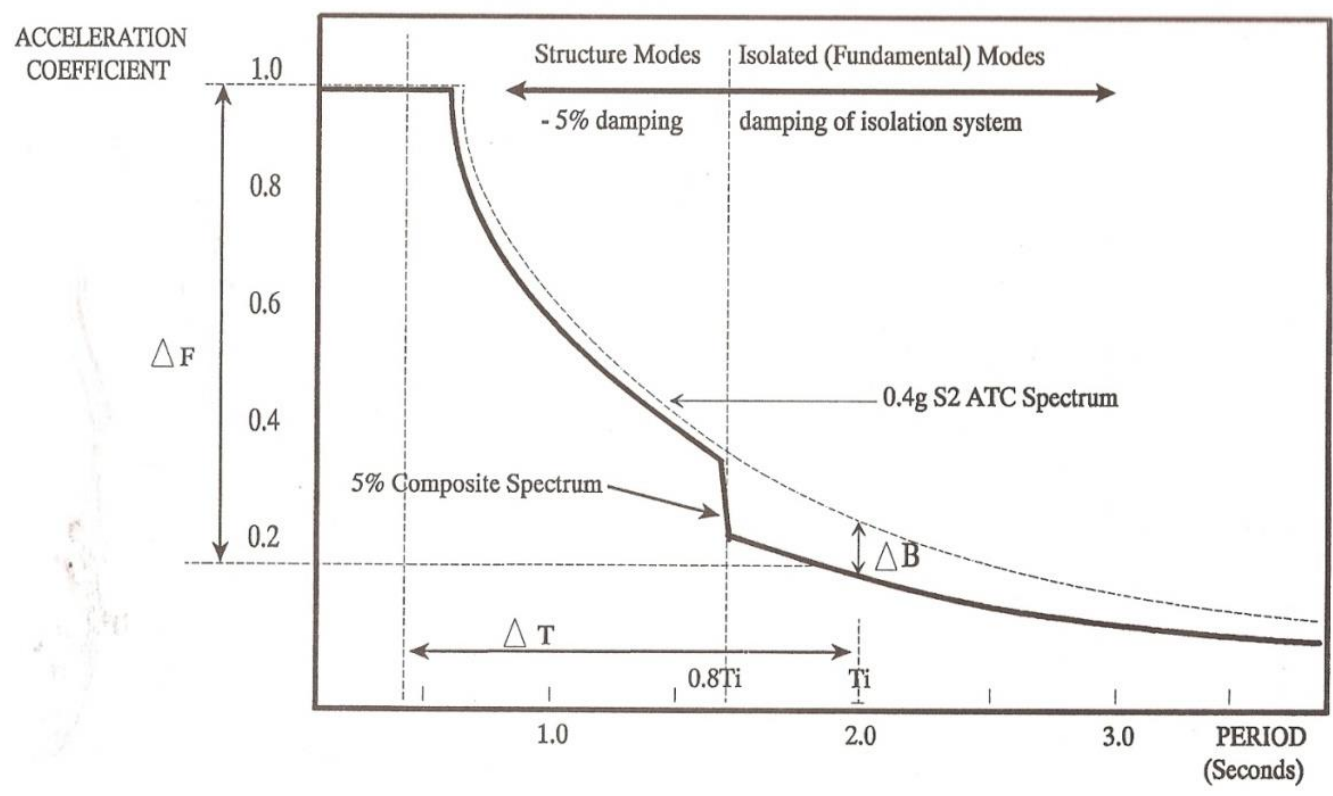

Figure 2. The AASHTO Acceleration spectrum. 
effective period, $T_{\text {eff }}$, which originates from the non-existing, $K_{\text {eff }}$, is silently upgraded with unsubstantiated liberty to a real physical quantity-that is the time needed for the isolated structure to complete one cycle of vibration. This technically weak concept is rooted to such an extent in the profession that several documents show values of $T_{\text {eff }}$ with superficial precision up to two decimal digits. Part of the motivation of this study is to investigate to whatextent the effective period, $T_{\text {eff }}$, may express the oscillatory response of a system with bilinear behavior.

\subsection{Simple Geometric Relations}

With reference to Fig. 1 (left) one can derive via the use of similar triangles a relation between the effective stiffness, $K_{\text {eff }}$ and the first slope of the bilinear model, $K_{1}$.

$$
K_{e f f}=K_{1} \frac{1+\alpha(\mu-1)}{\mu}
$$

and in terms of periods equation (4) gives

$$
T_{e f f}=T_{1} \sqrt{\frac{\mu}{1+\alpha(\mu-1)}}
$$

In the above equations, $\mu=u_{\max } / u_{y}$ is the displacement ductility and $\alpha=K_{2} / K_{1}$ is the second-to-the-first stiffness ratio. Equations (4) and (5) are well known in the literature (Hwang and Sheng (1993); (1994) and references reported therein). They are popular geometric relations which are valid for any value of the parameters $K_{1}, \alpha$ and $\mu$. Nevertheless, while the expression given by equation (5) is geometrically correct, its physical value remains feeble since there is no physical argument that associates the results of equation (5) with the vibration period of mass supported on a bilinear hysteretic system.

Fig. 3 plots with a solid line the values of the period shift, $T_{\text {eff }} / T_{1}$, as given by equation (5) as

a function of the displacement ductility $\mu$. The top-left plot of Fig. 3 is for values of $\alpha=K_{2} / K_{1}=0.15 \approx 1 / 6.5$, which is the value of $\alpha$ recommended by the New Zealand

Ministry of Works and Development (NZMWD 1983) for lead rubber bearings. Fig. 3 (bottom-left) plots the results of equation (5) for the widely used value of $\alpha=0.05$ (Hwang and Sheng 1993;1994); while, Fig. 3 (right) plots the results of equations (5) when spherical

sliding bearings are used. With reference to Fig. $1, K_{1}=\left(Q+K_{2} u_{y}\right) / u_{y}$ and therefore,

$$
\frac{1}{\alpha}=\frac{K_{1}}{K_{2}}=\frac{Q}{K_{2} u_{y}}+1=\frac{Q}{m} \frac{1}{u_{y}} \frac{T_{2}^{2}}{4 \pi^{2}}+1
$$

For a typical spherical sliding bearing $Q / m=0.05 g, u_{y}=0.25 \mathrm{~mm}=0.00025 \mathrm{~m}$ (Mokha et al. 1990, Constantinou et al. 1990), $T_{2}=2 \pi \sqrt{R / g}=2.5 \mathrm{~s}$, equation (6) yields a value of $\alpha=0.0032$. In the interest of completeness Table 1 offers the values of $\alpha=K_{2} / K_{1}$ for the typical values of strength, $Q / m$, second period, $T_{2}$, and values of yield displacement $u_{y}$ ranging from spherical sliding bearings to lead rubber bearings. Fig. 3 shows that regardless of the value of, $\alpha=K_{2} / K_{1}$, the period shift, $T_{\text {eff }} / T_{1}$, eventually tends asymptotically to the value $T_{2} / T_{1}=1 / \sqrt{\alpha}$ as the value of the ductility $\mu$ increases. This asymptotic trend in association that in spherical sliding bearings (SSB) the ductility $\mu$ is very large was probably the reason that several review type publications and textbooks (Naeim 2001, Naeim and Kelly 1999) abandoned the concept of the effective period, $T_{e f f}=2 \pi \sqrt{m / K_{e f f}}$ and they introduce, to the surprise of the non-expert, that for SSB, 
Lead Rubber Bearings
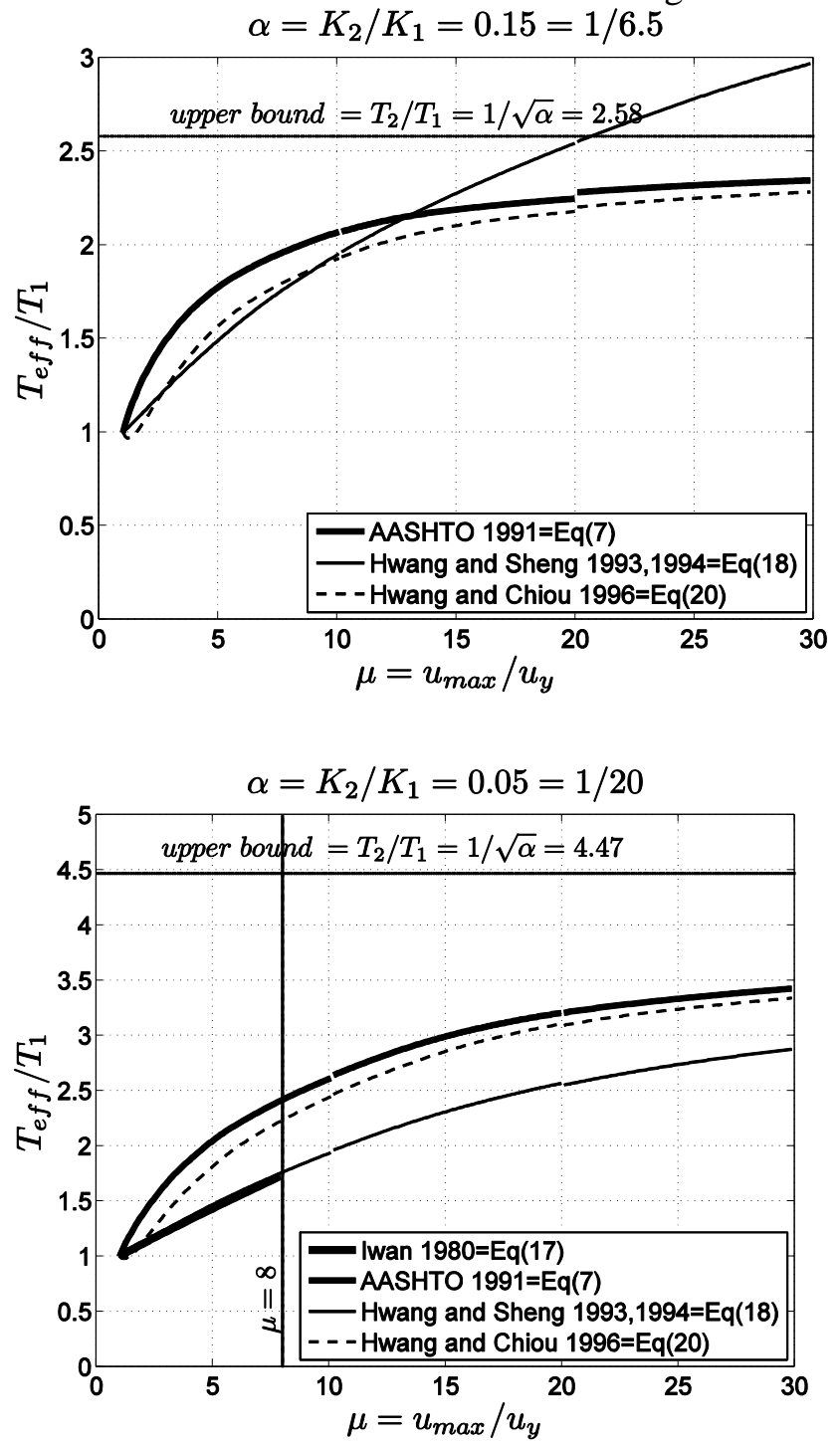

Spherical Sliding Bearings
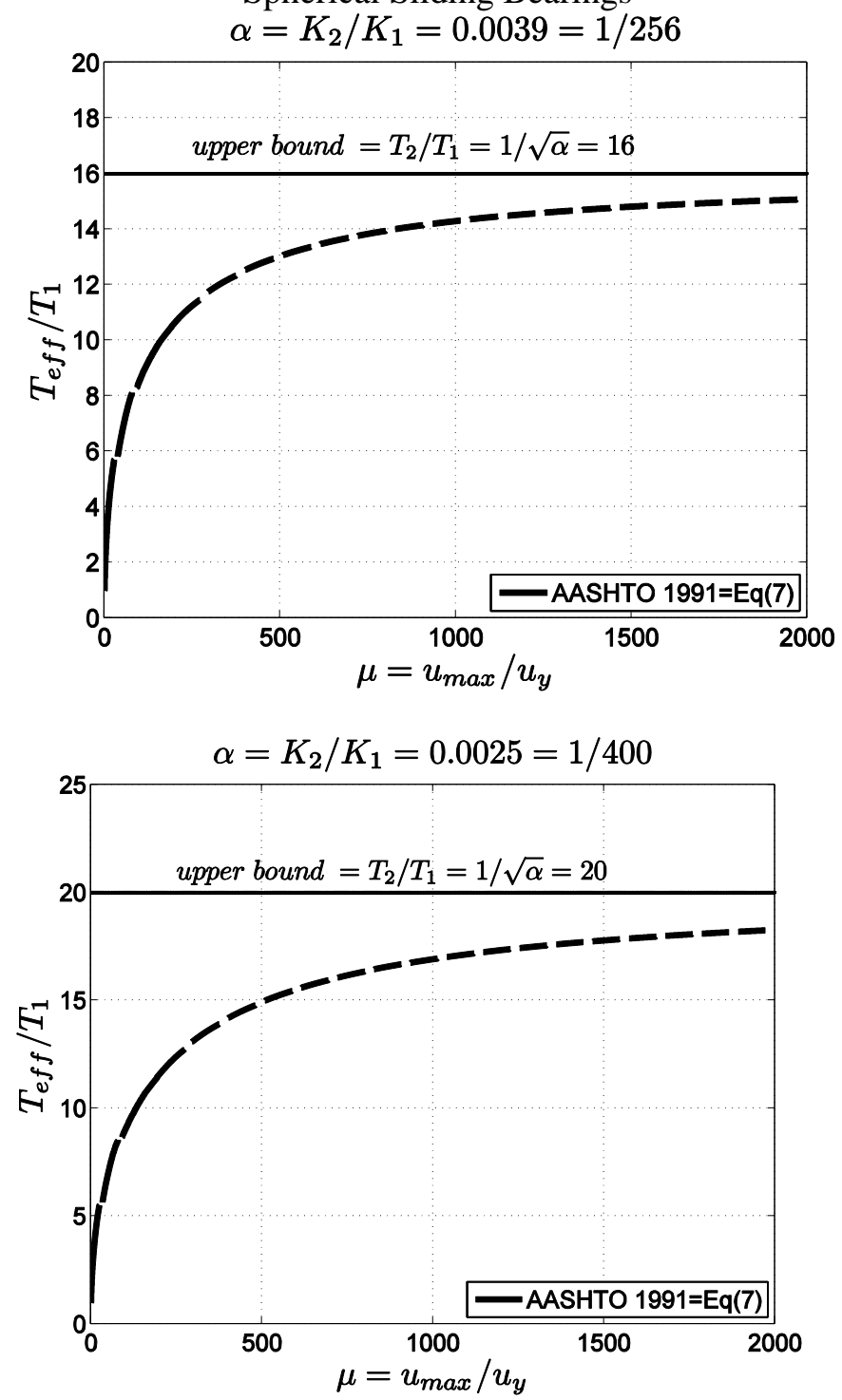

Figure 3. Values of the period shift, $T_{\text {eff }} / T_{1}$, as a function of the displacement ductility $\mu=u_{\max } / u_{y}$ as they result from similar triangles and other approximate expression presented in the literature.

Table 1. Values of $\alpha=K_{2} / K_{1}$ for typical values of isolation strength, $Q / m$ and yield displacement, $u_{y}$.

\begin{tabular}{|c|c|c|c|c|c|c|c|c|c|c|c|c|}
\hline & & & & & & $\alpha=$ & $/ K_{1}$ & & & & & \\
\hline & & $Q / m$ & $=0.03 g$ & & & $Q / m$ & $0.05 g$ & & & $Q / m=$ & $0.07 g$ & \\
\hline & & $u_{y}$ & $\mathrm{~cm})$ & & & $u_{y}$ & cm) & & & $u_{y}($ & cm) & \\
\hline$T_{2}(s)$ & 0.025 & 0.5 & 1.0 & 2.0 & 0.025 & 0.5 & 1.0 & 2.0 & 0.025 & 0.5 & 1.0 & 2.0 \\
\hline 2.0 & 0.0083 & 0.1435 & 0.2509 & 0.4012 & 0.0050 & 0.0913 & 0.1674 & 0.2867 & 0.0036 & 0.0670 & 0.1256 & 0.2231 \\
\hline 2.5 & 0.0053 & 0.0968 & 0.1766 & 0.3001 & 0.0032 & 0.0604 & 0.1140 & 0.2046 & 0.0023 & 0.0439 & 0.0842 & 0.1552 \\
\hline 3.0 & 0.0037 & 0.0693 & 0.1296 & 0.2295 & 0.0022 & 0.0428 & 0.0820 & 0.1516 & 0.0016 & 0.0309 & 0.0600 & 0.1132 \\
\hline 3.5 & 0.0027 & 0.0519 & 0.0986 & 0.1795 & 0.0016 & 0.0318 & 0.0616 & 0.1160 & 0.0012 & 0.0229 & 0.0448 & 0.0857 \\
\hline
\end{tabular}


$T_{I}=T_{2}=2 \pi \sqrt{R / g}$-not $T_{\text {eff }}$. Assuming a common inelastic displacement, $u_{\text {max }}$, for lead rubber and spherical sliding bearings, Fig. 3 shows that the effective period $T_{\text {eff }}$ as offered by equation (5) approaches the value of $T_{2}$ in a comparable way (when we look to the corresponding values of ductilities for LRB and SSB). Accordingly the question that rises is whether $T_{2}$ may also replace $T_{e f f}$ in the case of lead rubber bearings as well. Part of the scope of this paper is to offer an answer to this question.

Returning now to equation (4), the reader recognizes that as the yield displacement decreases, equation (4) combines very large $\left(K_{1}\right.$ and $\mu$ ) and very small $(\alpha)$ numbers. At the limiting case of a spherical sliding bearing, the first stiffness $K_{1}=\frac{Q}{u_{y}}+\alpha$-that is the elasticity of the teflon layer of the articulated slider before sliding occurs, is a very large quantity and totally indifferent to the design engineer while the corresponding value of $\alpha=K_{2} / K_{1}$ is a very small number as shown in Table 1.

Accordingly, the reader shall recognize that as the value of the ductility, $\mu=\frac{u_{\max }}{u_{y}}$, of

the bilinear system increases, equations (4) and (5) while they remain correct as geometric relations, their engineering value becomes marginal. What is much more interesting is to multiply and derive equation (4) by the second slope of the system, $K_{2}$; therefore, relating $K_{\text {eff }}$ and $K_{2}$ via the equation

$$
K_{e f f}=K_{2} \frac{1+\alpha(\mu-1)}{\alpha \mu}
$$

In terms of periods equation (7) becomes

$$
T_{\text {eff }}=T_{2} \sqrt{\frac{\alpha \mu}{1+\alpha(\mu-1)}}
$$

In studying the response of isolated structures, the displacement ductility $\mu=\frac{u_{\max }}{u_{y}}$ may vary either because the maximum displacement, $u_{\max }$, increases or because the yield displacement decreases when shifting from lead rubber bearings to spherical sliding bearings and in this case the ratio $\alpha=K_{2} / K_{1}$ varies as well. Accordingly, the behavior of equation (8) as the value of the ductility increases depends on the values that the product $\alpha \mu$ assumes (not just the values of $\mu$ ). Therefore, it is worth investigating the behavior of the product $\alpha \mu$ as $\mu$ increases.

With reference to Fig. $1, K_{1} u_{y}=Q+K_{2} u_{y}$; therefore,

$$
\frac{K_{2}}{\alpha} u_{y}=Q+K_{2} u_{y}
$$

Dividing equation (9) by the elastic force that develops in the isolation system $=K_{2} u_{\max }$, it yields

$$
\alpha \mu=\frac{1}{\frac{Q}{K_{2} u_{\max }}+\frac{1}{\mu}}
$$

And 


$$
\lim _{\text {forl arge } \mu} \alpha \mu=\frac{K_{2} u_{\text {max }}}{Q}
$$

Equation (11) shows that for large values of the displacement ductility, $\mu=\frac{u_{\max }}{u_{y}}$, the term, $\alpha \mu$, becomes a constant equal to the ratio of the elastic forces that develop in the isolation system, $K_{2} u_{\max }$, to the strength of the isolation system, $Q$. Accordingly, for large values of $\mu$ and after taking that $1-\alpha \approx 1$ equation (8) gives

$$
T_{\text {eff }}=\frac{1}{\sqrt{1+\frac{Q}{K_{2} u_{\max }}}} T_{2}
$$

When $\frac{Q}{K_{2} u_{\max }}<1$, equation (11) can be expanded into a Taylor series,

$$
\frac{T_{e f f}}{T_{2}}=1-\frac{1}{2} \frac{Q}{K_{2} u_{\max }}+\frac{3}{8}\left(\frac{Q}{K_{2} u_{\max }}\right)^{2}+\ldots
$$

In the event that one insists on using the concept of the effective period, $T_{\text {eff }}$, equation (12) (or 15) has much more physical meaning than equation (5) (or 10) since the displacement ductility, $\mu$, is a quantity of marginal interest in seismic isolation.

Given the introduction of the effective stiffness $K_{\text {eff }}=\frac{F_{\max }}{u_{\max }}$ by the aforementioned design codes, the main motivation of this paper is to examine to what extent equation (5) (or equation 10) which is a geometric relation that has been derived solely from similar triangles reflects indeed a physical reality-that is whether it expresses to a satisfactorily extent the oscillatory character of a bilinear system.

\subsection{The work of Iwan and Gates (1979) and Iwan (1980)}

Early theoretical work of the effective period and damping of stiffness-degrading structures was presented by Iwan and Gates (1979). The hysteretic model examined by Iwan and Gates (1979) is a collection of linear elastic and Coulomb slip elements which can approximate the phenomenon of cracking, yielding and crushing. A special case of their hysteretic model is the bilinear model that is of interest in this study. What is important to emphasize is that the Iwan and Gates (1979) study was motivated by the yielding response of traditional concrete and steel structures where the initial elastic stiffness, $K_{1}$, is a dominant parameter of the model; while, the displacement ductility assumes single digit values (say $\mu \leq 8$ ). Iwan and Gates (1979) observed that the average inelastic response spectra resemble the linear response spectra except for a translation along an axis of constant spectral displacement. The above observation was a major contribution at that time for it indicates that the effective period of each corresponding linear system would be of some constant multiple of the first period of the hysteretic system.

$$
T_{e f f}=C T_{1}
$$

Equation (14) is similar to equation (5); however, in the work of Iwan and Gates (1979) the constant, $C$, appearing in equation (14) is not an outcome from similar triangles (which result by assuming that $K_{\text {eff }}$ is the slope of the line that connects the axis origin with the point on the backbone curve where we anticipate the maximum displacement to occur), but is the outcome from minimizing the root mean square (RMS) of the difference between the spectral displacements of a bilinear system and a family of potentially equivalent linear systems. 
In a subsequent publication (Iwan (1980)), the period shift, $T_{\text {eff }} / T_{1}$, was graphed as a function of the ductility, $\mu$. The least square $\log -\log$ fit of these data resulted for a bilinear system with, $\alpha=K_{2} / K_{1}=0.05$, the following expression

$$
T_{\text {eff }}=\left[1+0.121(\mu-1)^{0.939}\right] T_{1}, \mu \leq 8
$$

It is worth mentioning that the work of Iwan and Gates (1979) and Iwan (1980) examined bilinear systems which exhibit values of displacement ductility up to $\mu=8$.

Fig. 3 (bottom-left) plots with a heavy solid line the values of the period shift, $T_{\text {eff }} / T_{1}$, as offered by equation (15) for $\alpha=0.05$ and up to values of ductility, $\mu=8$. These values are compared with the results from AASHTO (1991) geometric relation given by equation (5). As indicated earlier, the minimization procedure presented by Iwan and Gates (1979) and Iwan (1980) results an appreciably smaller period shift, $T_{\text {eff }} / T_{1}$, that what is predicted by the geometric relation given by equation (5).

\subsection{The work of Hwang and Shang (1993), (1994) and Hwang and Chiou (1996)}

While the work of Iwan and Gates (1979) investigated the effective period and damping of a bilinear system that approximates the nonlinear behavior of traditional concrete and steel structures (moderate values of the displacement ductility, $\mu<8$ ); Hwang and Sheng (1993); (1994) investigated the effective period of the bilinear system that approximates the nonlinear behavior of lead rubber isolation bearings where the displacement ductility can reach the value of $\mu=25$ or greater.

Initially, Hwang and Sheng (1993); (1994) argued that the effective stiffness and the associated effective period of the bilinear system as suggested by the 1991 AASHTO Guide Specifications for seismic isolation (eq.(5)) is an unrealistic representation of what happens in reality and suggested the following expression for the effective period for all practical values of $\alpha=K_{2} / K_{1}$

$$
T_{\text {eff }}=\left\{1+\ln \left[1+0.13(\mu-1)^{1.137}\right]\right\} T_{1}
$$

The predictions of equation (16) which was presented later as the Caltrans Method (Hwang and Chiou 1996) are also shown in Fig. 3(top-left) with a thin solid line.

Equation (16) as well as any empirical equation that attempts to offer an estimate on the effective period $T_{\text {eff }}$ shall also satisfy the constraint-that the proposed period $T_{\text {eff }}$ shall always be less than or equal to the period $T_{2}$ which corresponds to the second slope of the bilinear system. Accordingly,

$$
\frac{T_{e f f}}{T_{1}} \leq \frac{T_{2}}{T_{1}}=\frac{1}{\sqrt{\alpha}}
$$

Fig. 3 (top-left) shows that for $\alpha=K_{2} / K_{1}=0.15$ equation (16) proposed by Hwang and Sheng $(1993,1994)$ violates the physical constraint given by equation (17) for values $\mu>20$; whereas, Fig. 3 (bottom-left) shows that for $\alpha=K_{2} / K_{1}=0.05$ equation (16) offers an isolation period that is even shorter than the isolation period which results from the geometric relation given by equation (5) adopted by AASHTO 1991.

In a subsequent publication, Hwang and Chiou 1996 proposed a refined model for lead rubber bearings where the effective period is offered by the following equation:

$$
T_{\text {eff }}=\sqrt{\frac{\mu}{1+\alpha(\mu-1)}}\left(1-0.737 \frac{\mu-1}{\mu^{2}}\right) T_{1}
$$

Equation (18) is merely the geometric relation adopted by AASHTO (1991) given by equation (5) modified by the multiplication factor $1-0.737(\mu-1) / \mu^{2}$. The predictions of 
equation (18) (dashed line) are also offered in Fig. 3 (left) which is relevant to lead rubber bearings. Note that the predictions of equation (18) follow very closely the geometric relation adopted by AASHTO (1991), revealing that the multiplication factor $1-0.737(\mu-1) / \mu^{2}$ has a minor effect. The proximity of equation (18) that was finally proposed by Hwang and Chiou (1996) to the predictions of equation (5) adopted by AASHTO (1991) leaves the reader perplexed given the initial comments of Hwang and Sheng (1993); (1994) that the effective period of the bilinear system as suggested by the 1991 AASHTO Guide Specifications is an unrealistic representation of what happens in reality.

\section{The Relative Importance of the Parameters of the Bilinear Model Associated with the Behavior of Seismic Isolation Bearings}

Before proceeding with the evaluation of the engineering merit of the "effective period" of the bilinear system, in this section we discuss the relative significance of the parameters of the bilinear system. With reference to Fig. 1 (left) the bilinear model is fully described with any three of the five parameters shown in Fig. 1, which are the strength, $Q$, the initial stiffness, $K_{1}$, the yield force, $F_{y}$, the yield displacement, $u_{y}$, and the second stiffness, $K_{2}$.

The main difference between the behavior of bilinear isolation bearings and the behavior of traditional steel and reinforced concrete structures which also exhibit a bilinear behavior is in the values of strength and peak inelastic deformation. Isolation bearings have intentionally lower strength (say $0.03 \leq Q / m g \leq 0.09$ ); and therefore, experience large values of inelastic displacements. Accordingly, the behavior of bilinear isolation bearings is primarily controlled by the strength, $Q$, and the second stiffness, $K_{2}$; while, the yield displacement, $u_{y}$, and the associated first stiffness, $K_{1}$ have marginal significance. The marginal significance of the yield displacement, $u_{y}$, in the peak response of a bilinear isolation system has been shown in the past via parametric studies (Makris and Chang (2000)) and subsequently through formal dimensional analysis (Makris and Black (2004), Makris and Vassiliou (2011)). Given the marginal significance of the yield displacement, $u_{y}$, the displacement ductility $\mu=u_{\max } / u_{y}$ is also of marginal interest in seismic isolation and should be avoided as a dimensionless quantity (see equations (12) or (13)).

Now while the design codes on seismic isolation do not state explicitly the marginal significance of the first stiffness, $K_{1}$, the iterative procedure proposed by the design codes introduced in the previous section to converge on the effective stiffness, $K_{\text {eff }}$, involves only the strength, $Q$, and the second stiffness, $K_{2}$ (the first stiffness, $K_{1}$, and the yield displacement, $u_{y}$, are immaterial in estimating $u_{\max }$ ). Accordingly, the behavior of the bilinear model is described in a robust way by the controlling parameters, $Q$ and $K_{2}$ together with one of the marginal parameters $K_{1}, u_{y}$ or $F_{y}$. In this study, for the third parameter we select $u_{y}$. Its value ranges from $0.25 \mathrm{~mm}$ (spherical sliding bearing, Mokha et al. (1990)) up to $2 \mathrm{~cm}$ or even higher (for lead rubber bearings).

\section{Free-Vibration Period of a Bilinear System}

Our evaluation on the engineering merit of the effective period, $T_{\text {eff }}$, commences with the free-vibration response analysis of a mass $m$ supported on a mechanical system with bilinear behavior. Given that the bilinear behavior is fully described by the normalized strength, $Q / \mathrm{m}$, the normalized second stiffness $K_{2} / m$ and the yield displacement, $u_{y}$, the free vibration period-that is the isolation period, $T_{I}$ is a function of 


$$
T_{I}=f\left(\frac{Q}{m}, u_{y}, \frac{K_{2}}{m}\right)
$$

The four variables appearing in equation $T_{I}=[T], \quad \frac{Q}{m}=[L][T]^{-2}, \quad u_{y}=[L], \quad \frac{K_{2}}{m}=[T]^{-2}$ involve only two reference dimensions; that of length $[L]$ and time $[T]$. According to Buckingham's $\Pi$-theorem, the number of independent dimensionless products that describe the problem is the number of total physical variables $=4$ minus the number of reference dimensions $=2$. Therefore, the number of dimensionless products that describe the problem is $4-2=2$. Since the repeating variables need to have independent dimensions, the choice for the repeating variable is the period associated with the second slope; $T_{2}=2 \pi \sqrt{m / K_{2}}=[T]$ and the yield displacement $u_{y}=[L]$. Consequently, the two dimensionless products are $\Pi_{T}=T_{I} / T_{2}=T /\left(2 \pi \sqrt{m / K_{2}}\right)$ and $\Pi_{Q}=(Q / m) \times\left(1 / u_{y}\right) \times\left(m / K_{2}\right)=Q /\left(K_{2} u_{y}\right)$. With the two dimensionless $\Pi$-products established equation $(19)$ reduces to

$$
\frac{T_{I}}{2 \pi \sqrt{\frac{m}{K_{2}}}}=\varphi\left(\frac{Q}{K_{2} u_{y}}\right) \Rightarrow T_{I}=\varphi\left(\frac{Q}{K_{2} u_{y}}\right) T_{2}
$$

Equation (20) indicates that the free-vibration period of the bilinear system is equal to the period associated with the second slope, $T_{2}=2 \pi \sqrt{m / K_{2}}$, while being modified by some function $\varphi\left(Q /\left(K_{2} u_{y}\right)\right)$. In order to find the expression of the function $\varphi\left(Q /\left(K_{2} u_{y}\right)\right)$ we conduct a series of numerical runs to compute the free-vibration response of the bilinear system. A rigid mass supported on bilinear bearings with second slope $K_{2}$ is set away from equilibrium at a given initial displacement (say $u_{0}=20 \mathrm{~cm}, 30 \mathrm{~cm}$, and $40 \mathrm{~cm}$ ) and zero initial velocity and is let free to undergo free vibration.

\subsection{Fourier Analysis}

In our investigation only runs where a full cycle or more was completed were retained and the free-vibration period was defined as the period where the peak value in the Fourier spectrum happens. Fig. 4 shows selective force displacement loops of the bilinear system under free vibration response together with the corresponding Fourier spectra. The vibration period of the system, $T_{I}$, is extracted at the period where the maximum of the Fourier spectrum happens. Fig. 5 (left) plots the computed isolation period, $T_{I}$, normalized to the period associated with the second slope, $T_{I} /\left(2 \pi \sqrt{m / K_{2}}\right)$, as a function of the dimensionless product $\Pi_{Q}=Q /\left(K_{2} u_{y}\right)=K_{1} / K_{2}-1=1 / \alpha-1$. Regression analysis from all the data yields a mean value for $T_{I} / T_{2}=0.99$, indicating that the function $\varphi\left(Q /\left(K_{2} u_{y}\right)\right)$ appearing in equation (19) is merely a constant with value, $\varphi=0.99 \approx 1$. The remarkable finding from this analysis is that the vibration period of a bilinear isolation system is precisely the period associated with the second slope $=T_{2}$. Furthermore, the standard deviation of the computed values from the mean value $T_{I} / T_{2}=0.99$ is very small, $S D=0.045$, showing that the computed data are strongly correlated with the vibration period $T_{2}$. Fig. 5 (right) plots the computed isolation period, $T_{I}$, from the Fourier analysis normalized to the effective period, $T_{\text {eff }}$. In this calculation the effective stiffness, $K_{\text {eff }}=F_{\max } / u_{0}$ was computed as the ratio of the peak force $F_{\max }$, that develops at the peak initial displacement, $u_{0}$, prior to the initiation 

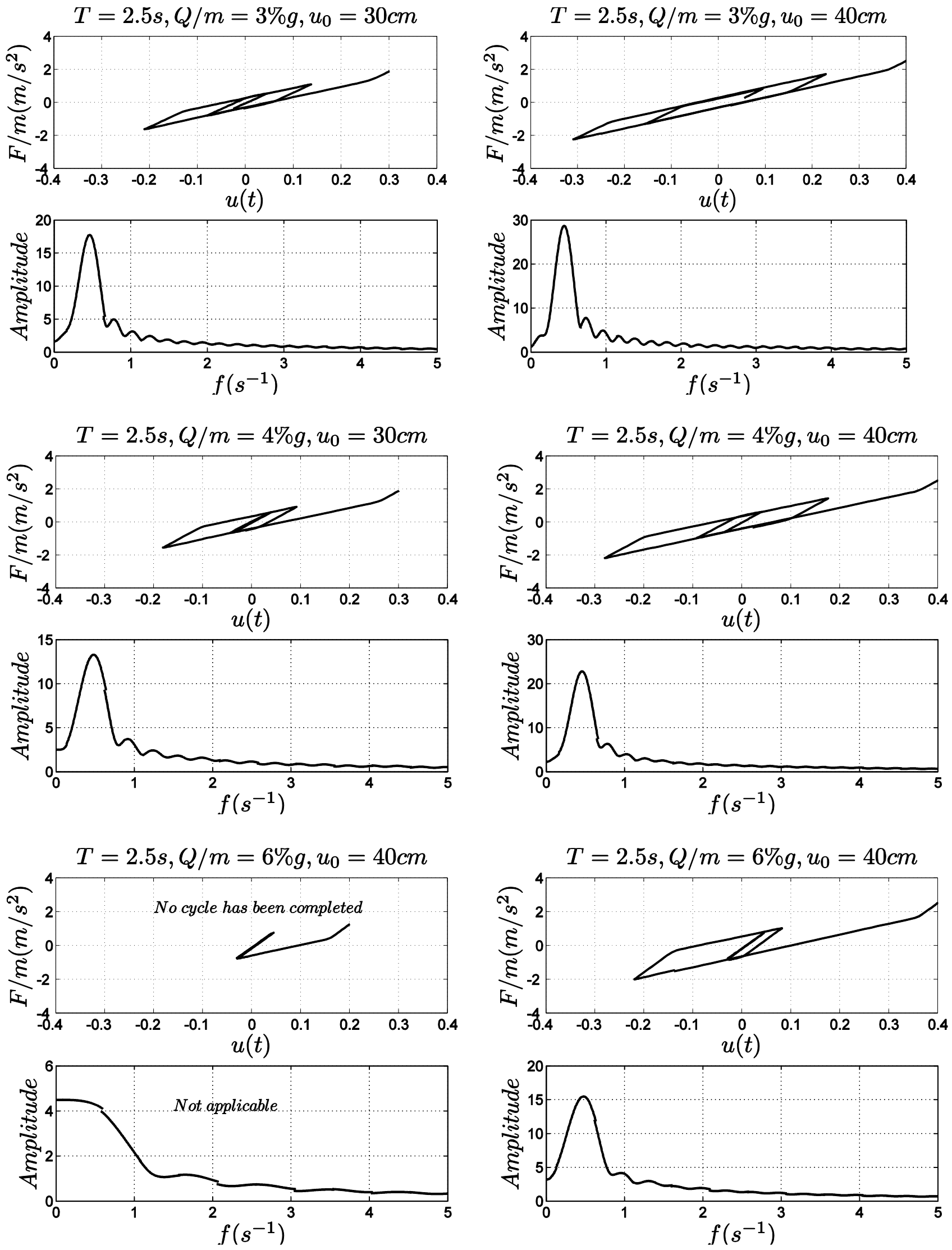

Figure 4. Selective force-displacement loops from the free vibration response of bilinear isolation systems $\left(T_{2}=2.5 \mathrm{~s}\right)$ and the associated Fourier spectra. 

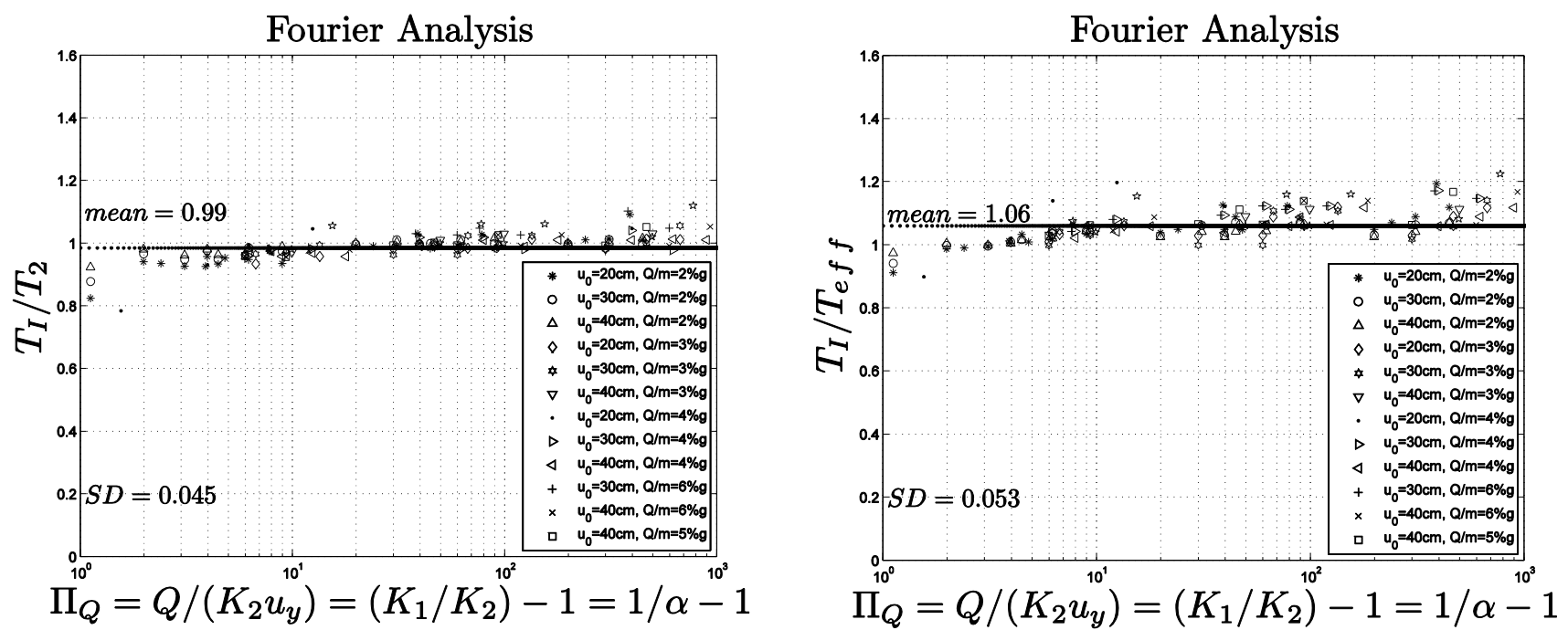

Figure 5. Values of the vibration period of bilinear isolation systems during free vibrations extracted with Fourier analysis

of the motion which is a calculation that selects the longest possible $T_{\text {eff }}$. The regression analysis from all data yields a mean value for $T_{I} / T_{\text {eff }}=1.06$, and a very small standard deviation, $S D=0.053$, indicating that $T_{\text {eff }}$ is a dependable approximation of the vibration period of the bilinear system; nevertheless, the period associated with the second slope, $T_{2}$ is an even better approximation (see Fig. 5 left).

\subsection{Wavelet Analysis}

The result offered by Fig. 5-that the free-vibration period of the bilinear system is essentially the period associated strictly with the second slope, is further confirmed by analyzing the free-vibration response histories with wavelet analysis. Over the last two decades, wavelet transform analysis has emerged as a unique new time-frequency decomposition tool for signal processing and data analysis. There is a wide literature available regarding its mathematical foundation and its applications (Mallat 1999, Addison 2002 and references reported therein). Wavelets are simple wavelike functions localized on the time axis. For instance, the second derivative of the Gaussian distribution, $e^{-t^{2} / 2}$, known in seismology literature as the symmetric Ricker wavelet (Ricker 1943; 1944; and widely referred as the "Mexican Hat" wavelet, Addison 2002),

$$
\psi(t)=\left(1-t^{2}\right) e^{-t^{2} / 2}
$$

is a widely used wavelet. Similarly the time derivative of equation (21) or a one cycle cosine function are also wavelets. A comparison on the performance of various symmetric and antisymmetric wavelet to fit acceleration records is offered in Vassiliou and Makris (2011). In order for a wavelike function to be classified as a wavelet, the wavelike function must have: (a) finite energy,

$$
E=\int_{-\infty}^{+\infty}|\psi(t)|^{2} d t<\infty
$$

and (b) a zero mean. In this work we are merely interested to achieve a local matching of the response history of a bilinear system with a wavelet that will offer the best estimates of period, $T_{I}$. Accordingly, we perform a series of inner products (convolutions) of the acceleration response history of the bilinear system, $\ddot{u}(t)$ with the wavelet $\psi(t)$ by manipulating the wavelet through a process of translation (i.e. movement along the time axis) and a process of dilation-contraction (i.e. spreading out or squeezing of the wavelet) 

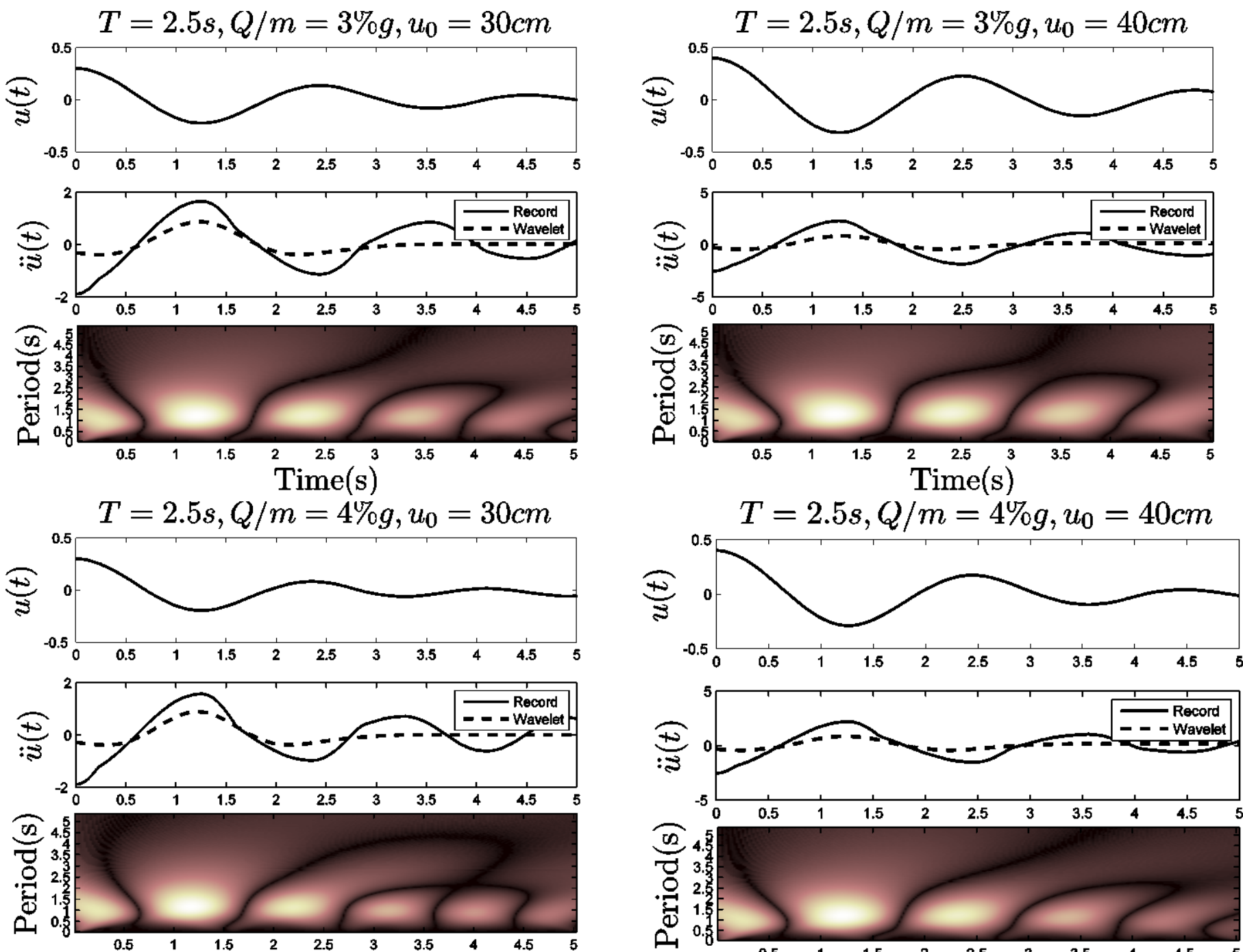

Time(s)
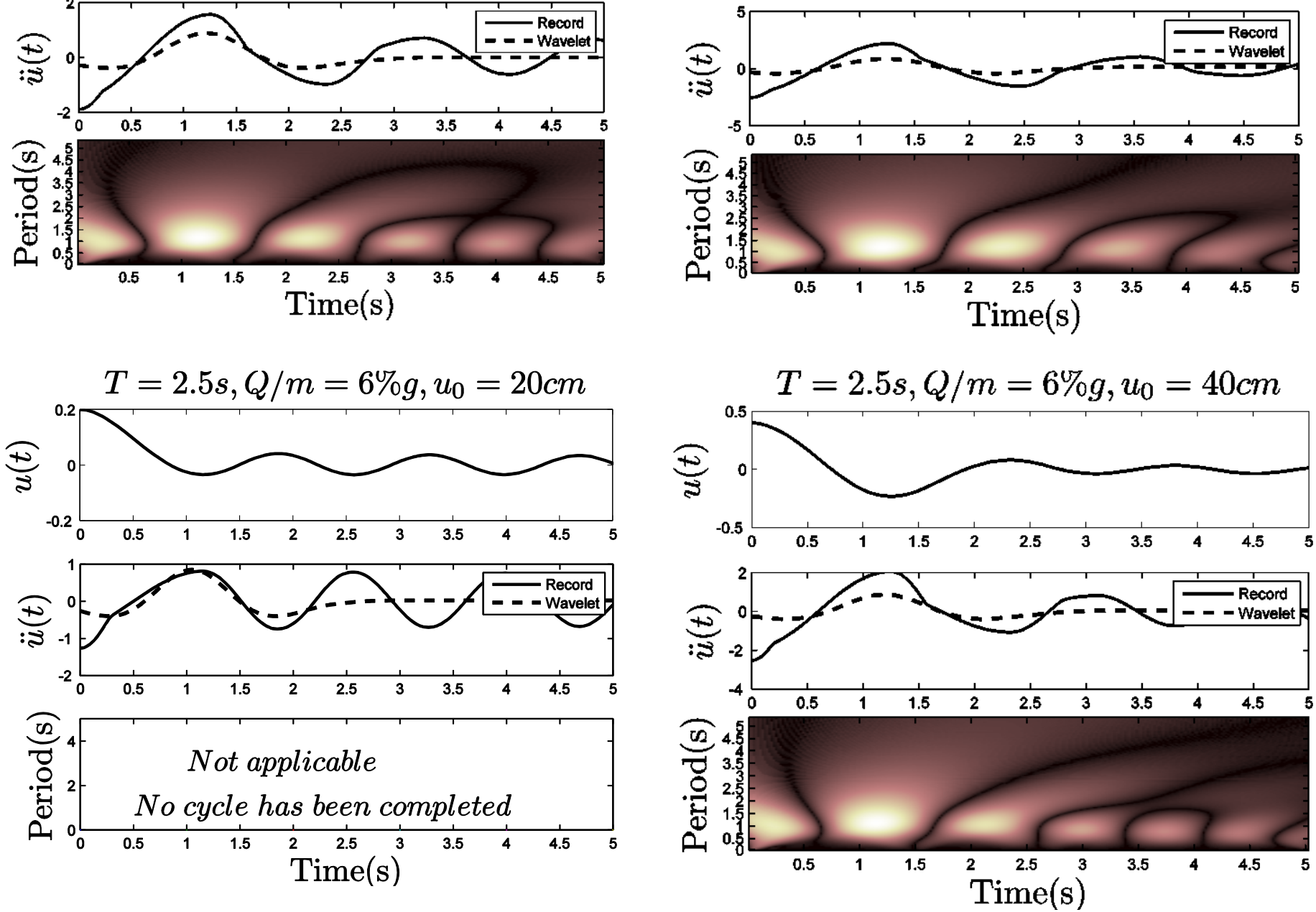

Figure 6. Matching the free vibration acceleration histories of bilinear systems $\left(T_{2}=2.5 \mathrm{~s}\right)$ with symmetric Ricker wavelets together with the associated scalograms. In a symmetric Ricker wavelet the period $T=\pi \sqrt{2} S$, where $S$ is the scale of the wavelet. 

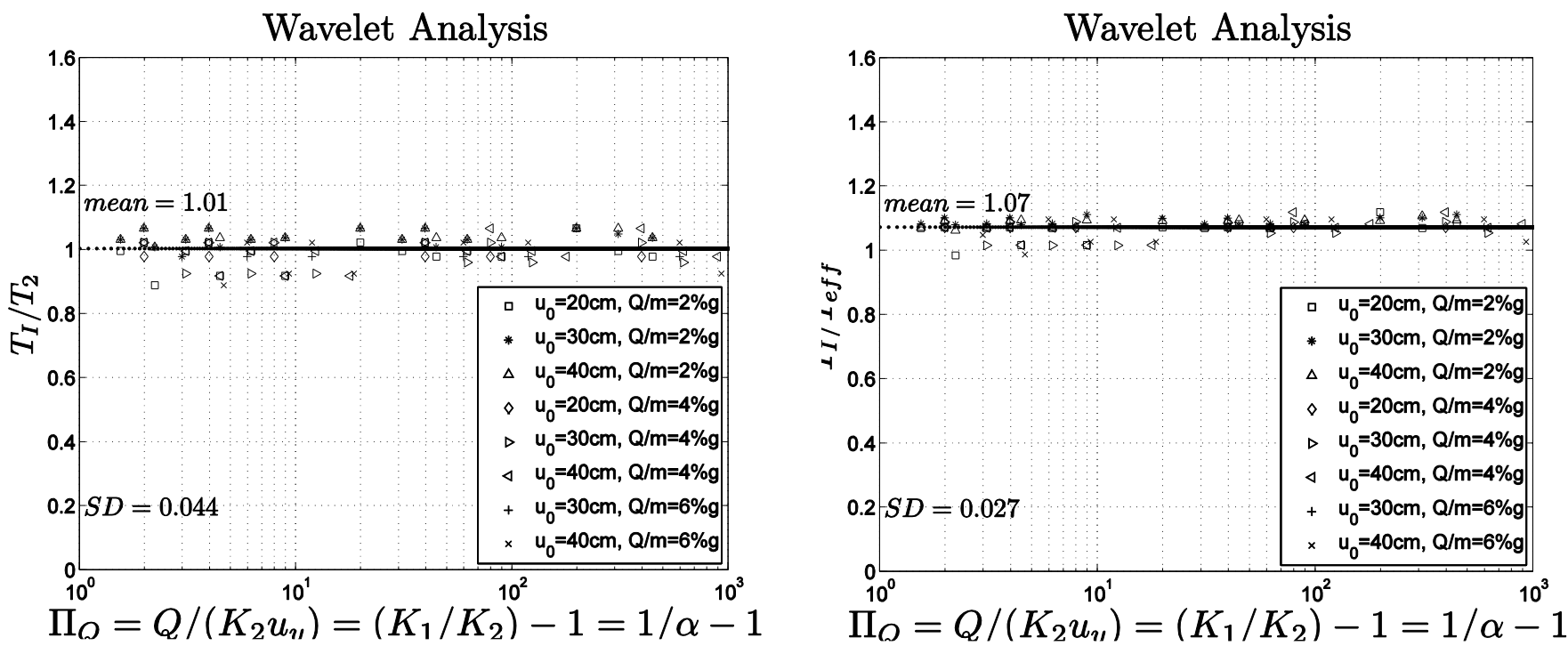

Figure 7. Values of the vibration period of bilinear isolation systems during free vibrations extracted with wavelet analysis.

$$
C(s, \xi)=w(s) \int_{-\infty}^{+\infty} \ddot{u}(t) \psi\left(\frac{t-\xi}{s}\right) d t
$$

The values of $s=S$ and $\xi=\Xi$ for which the coefficient, $C(s, \xi)$ becomes maximum offer the scale and location of the wavelet $w(s) \psi\left(\frac{t-\xi}{s}\right)$ that locally matches best the freevibration response history, $\ddot{u}(t)$. Equation (23) is the definition of the wavelet transform. The quantity $w(s)$ outside the integral in Equation (23) is a weighting function. Typically, $w(s)$ is set equal to $1 / \sqrt{s}$ in order to ensure that all daughter wavelets $\psi_{s, \xi}(t)=w(s) \psi\left(\frac{t-\xi}{s}\right)$ at every scale $S$ have the same energy. The same energy requirement among all the daughter wavelets $\psi_{s, \xi}(t)$ is the default setting in the MATLAB wavelet toolbox (2002) and what is used in this analysis. A detail analysis on the role of the weighting function in the definition of the wavelet transform is presented in Vassiliou and Makris (2011). Each set of plots in Fig. 6 show the best matching Ricker wavelet on selected free vibration responses of bilinear isolation systems (center); while, the associated scalogram (bottom) shows contours of the value of $C(s, \xi)$ as defined by equation (23) for all locations, $t$, and all scales, $\xi$. The maximum value $C(s, \xi)$ happens at the most bright location of the contour.

Similar to Fig. 5 (left), Fig. 7(left) plots the computed isolation period $T_{I}$ normalized to the period associated with the second slope $T_{I} /\left(2 \pi \sqrt{m / K_{2}}\right)$, as a function of the dimensionless product $\Pi_{Q}=Q /\left(K_{2} u_{y}\right)=K_{1} / K_{2}-1=1 / \alpha-1$ as extracted with wavelet analysis. In Fig. 7 the period of free vibration of the bilinear system has been extracted with the symmetric Ricker wavelet. Regression analysis from the data yields a mean value for $T_{I} / T_{2}=1.01$ and a small standard deviation, $S D=0.044$ which confirms in a decisive manner that the function $\varphi$ appearing in equation (5) is a constant with $\varphi=1$. Fig. 7 (right) shows that the regression analysis from all data yields a mean value for $T_{I} / T_{\text {eff }}=1.06$ and a small standard deviation, $S D=0.027$, indicating that $T_{\text {eff }}$ as results from $K_{\text {eff }}=F_{\max } / u_{0}$ is a good approximation of the vibration period of the bilinear system, nevertheless, the period 
associated with the second slope, $T_{2}$ is an even better approximation. Consequently, the results presented in Fig. 5 and 7 suggest that as long as a bilinear system completes one cycle under free vibration the period that prevails during its free vibration is $T_{2}$-that is the period associated with the second slope $K_{2}$; and this result is independent of the value of the dimensionless strength $\Pi_{Q}=\frac{Q}{K_{2} u_{y}}=\frac{1}{\alpha}-1$.

\section{Predominant Period of a Bilinear System under Forced Excitation}

\subsection{Pulse Excitation}

Our evaluation on the engineering merit of the effective period, $T_{\text {eff }}$, proceeds with the forced vibration response analysis of the bilinear system which is first subjected to a pulse excitation. The motivation for first using pulse excitations is to deal initially with coherent excitations that contain only a narrow frequency band. As excitation pulse we select the symmetric Ricker acceleration pulse (Ricker 1943;1944)

$$
\ddot{u}_{g}(t)=a_{p}\left[1-2 \pi^{2} \frac{\left(t-T_{p}\right)^{2}}{T_{p}{ }^{2}}\right] e^{-\pi^{2}\left(\frac{t-T_{p}}{T_{p}}\right)^{2}}
$$

which is a popular wavelike function to approximate the coherent pulse that appears in several recorded near source ground motions (Garini et al. 2010, Vassiliou and Makris 2011. The right hand side of equation (24) is merely the mother Ricker wavelet given by (21) which has been magnified with an acceleration amplitude $a_{p}$ dilated by a scale $s=\frac{\sqrt{2}}{2 \pi} T_{p}$ and translated by $T_{p}$. The forced vibration response of the bilinear system is fully described by the three parameter mentioned before which describe its bilinear behavior-that is the normalized strength, $\frac{Q}{m}$, the normalized second slope, $T_{2}=2 \pi \sqrt{\frac{m}{K_{2}}}$, and the yield displacement, $u_{y}$; together with the two parameters that describe the pulse excitation-that is the pulse acceleration, $a_{p}$, and the pulse period, $T_{p}$. Accordingly, the period that dominates the forced vibration response of the bilinear system -that is the isolation period, $T_{I}$, is a function of

$$
T_{I}=f\left(\frac{Q}{m}, u_{y}, T_{2}, a_{p}, T_{p}\right) .
$$

The six variables appearing in equation (26) $T_{I}=[T], Q / m=[L][T]^{-2}, u_{y}=[L]$ $T_{2}=2 \pi \sqrt{m / K_{2}}=[T], a_{p}=[L][T]^{-2}$ and $T_{p}=[T]$ involve again only two reference dimension that of length $[L]$ and time $[T]$; therefore, the number of dimensionless products

that describe the problem is $6-2=4$. As in the previous sections the choice for the repeating variables is the period associated with the second slope, $T_{2}=2 \pi \sqrt{m / K_{2}} \doteq[T]$ and the yield displacement, $u_{y}=[L]$. Based on this organization the dependent dimensionless product

$$
\Pi_{I}=\frac{T_{I}}{T_{2}}
$$

is a function only of the independent dimensionless products 


$$
\begin{gathered}
\Pi_{Q}=\frac{Q}{K_{2} u_{y}} \\
\Pi_{T}=\frac{T_{p}}{T_{2}} \\
\Pi_{a}=\frac{a_{p} T_{2}^{2}}{u_{y}}
\end{gathered}
$$

While the first two dimensionless products $\Pi_{Q}$ and $\Pi_{T}$ have a clear engineering significance, the engineering significance of $\Pi_{a}$ is not clear. Nevertheless, the quantity, $\Pi_{a} / \Pi_{Q}=4 \pi^{2} m a_{p} / Q$ which is the ratio of the inertia forces to the strength of the system has a clear engineering significance. Accordingly, the dimensionless product given by (29) is replaced with the dimensionless product

$$
\Pi_{a q}=\frac{1}{4 \pi^{2}} \frac{\Pi_{a}}{\Pi_{Q}}=\frac{m a_{p}}{Q},
$$

and equation (27) is reduced to

$$
\frac{T_{I}}{T_{2}}=\varphi\left(\frac{Q}{K_{2} u_{y}}, \frac{T_{p}}{T_{2}}, \frac{m a_{p}}{Q}\right) \Rightarrow T_{I}=\varphi\left(\frac{Q}{K_{2} u_{y}}, \frac{T_{p}}{T_{2}}, \frac{m a_{p}}{Q}\right) T_{2}
$$

As in the free vibration case, equation (31) indicates that the period which prevails during force vibration-that is the isolation period is equal to the period associated with the second slope $T_{2}=2 \pi \sqrt{m / K_{2}}$, while being modified by some function $\varphi\left(\frac{Q}{K_{2} u_{y}}, \frac{T_{p}}{T_{2}}, \frac{m a_{p}}{Q}\right)$.

The dynamic response of a mass, $m$, supported on isolation bearings with bilinear behavior as described in Fig. 1 is governed by

$$
\ddot{u}+\omega_{2}{ }^{2} u(t)+\frac{Q}{m} z(t)=-\ddot{u}_{g}(t)
$$

where $u(t)=$ relative to the ground displacement history, $\ddot{u}_{g}(t)=$ ground acceleration, $Q / m=$ specific strength, $\omega_{2}=2 \pi / T_{2}$ and $z(t)=$ hysteretic dimensionless quantity with $|z(t)| \leq 1$ that is governed by

$$
u_{y} \dot{z}(t)+\gamma|\dot{u}(t)| z(t)|z(t)|^{n-1}+\beta \dot{u}(t)|z(t)|^{n}-\dot{u}(t)=0
$$

The model given by equations (32) and (33) is the Bouc-Wen model (Wen 1975; 1976 in which $\beta, \gamma$ and $n$ are dimensionless quantities that control the shape of the hysteretic loop. Fig. 8 plots with a solid line the acceleration responses above isolators of two different isolation systems subjected to a Ricker pulse excitation shown below.

\subsubsection{Wavelet Analysis}

The identification of the vibration period of bilinear isolation systems subjected to pulse excitation is first achieved with the wavelet analysis introduced in the previous section. Fig. 8 (left) plots with a dashed line the best matching wavelet (Vassiliou and Makris 2011) on the acceleration response history above isolators with bilinear behavior. A number of bilinear systems and Ricker pulse excitations have been selected to cover a wide range of the dimensionless products given by equation (27), (28) and (30). Fig. 9 (left) plots the computed isolation period $T_{I}$ normalized to the period associated with the second period $T_{2}=2 \pi \sqrt{m / K_{2}}$ as a function of the dimensionless products 
$\Pi_{Q}=Q /\left(K_{2} u_{y}\right)=K_{1} / K_{2}-1=1 / \alpha-1$ as extracted with wavelet analysis in which the daughter wavelet is the Mavroeidis and Papageorgiou (M\&P) wavelet (Vassiliou and Makris 2011). Regression analysis from all data yields a mean value for $T_{I} / T_{2}=1.05$, indicating that the function $\varphi\left(\frac{Q}{K_{2} u_{y}}, \frac{T_{p}}{T_{2}}, \frac{m a_{p}}{Q}\right)$ appearing in equation (19) is a constant with value $\varphi=1.05$. At the same time it shall be recognized that the standard deviation now is $S D=0.082$ which is two times larger than the value of the standard deviation computed during the free vibration response (see Fig. 5 and 7). Fig. 9 (right) plots the computed vibration (isolation) period, $T_{I}$, with wavelet analysis normalized to the effective period, $T_{\text {eff }}$. In this calculation the effective stiffness, $K_{\text {eff }}=F_{\max } / u_{\max }$ was computed by finding the peak force developed at the peak inelastic displacement $u_{\max }$ identified during the response history and regression analysis from all data yields a mean value for $T_{I} / T_{\text {eff }}=1.15$ and a standard deviation $S D=0.076$. Accordingly, this analysis shows that $T_{\text {eff }}$ as given by equation (5) is a dependable approximation of the vibration period of the bilinear system; nevertheless, the period associated with the second slope, $T_{2}$, is an even better approximation.

\subsubsection{Time Domain Analysis}

Over the years, various powerful time domain methods have been developed and applied successfully. Perhaps, the most well known and powerful method in the system identification community is the Prediction Error Method (PEM).

It initially emerged from the maximum likelihood framework of Aström and Bohlin (1965) and subsequently was widely accepted via the corresponding MATLAB (2002) identification toolbox developed following the theory advanced by Ljung (1987); (1994); (2002). Fig. 8(right) shows the identification of the acceleration history above isolators of two bilinear isolation system with PEM when excited by the symmetric Ricker wavelets shown in the left of Fig. 8. The identification has been conducted only in the segment of the time history where the bearing deformation enters the second slope.

The engagement of the second slope is monitored with the dimensionless internal variable $z(t)$ which is governed by equation (33) and assumes the values $(+1,-1)$ when the bearing displacement, $u(t)>u_{y}$. The time histories of the $z(t)$ are also shown in Fig. 8 (right). Fig. 10 (left) plots the computed vibration (isolation) period $T_{I}$, with the prediction error method (PEM) normalized with the second period, $T_{2}$. While there are several data on the $T_{I} / T_{2}=1$ line in particular for large values of $\Pi_{Q}$, the data show a wider scattering than the data obtained with wavelet analysis (see Fig. 9) resulting a mean value for $T_{I} / T_{2}=0.89$ with a standard deviation $S D=0.160$. Fig. 10 (right) plots the computed vibration (isolation) period $T_{I}$, with PEM normalized to the effective period, $T_{\text {eff }}$. As in the wavelet analysis, in this calculation the effective stiffness $K_{\text {eff }}=F_{\text {max }} / u_{\max }$ was computed by finding the peak force developed at the peak inelastic displacement $u_{\max }$ identified during the response history and regression analysis from all data yields a mean value for $T_{I} / T_{\text {eff }}=0.97$ and a standard deviation $S D=0.164$. Accordingly, when the PEM is employed the period associated with $T_{\text {eff }}$ appears to be a better approximation.

\subsection{Earthquake Excitation}

Our evaluation on the engineering merit of the effective period, $T_{\text {eff }}$, continues with the forced vibration response analysis of the bilinear system when subjected to earthquake 

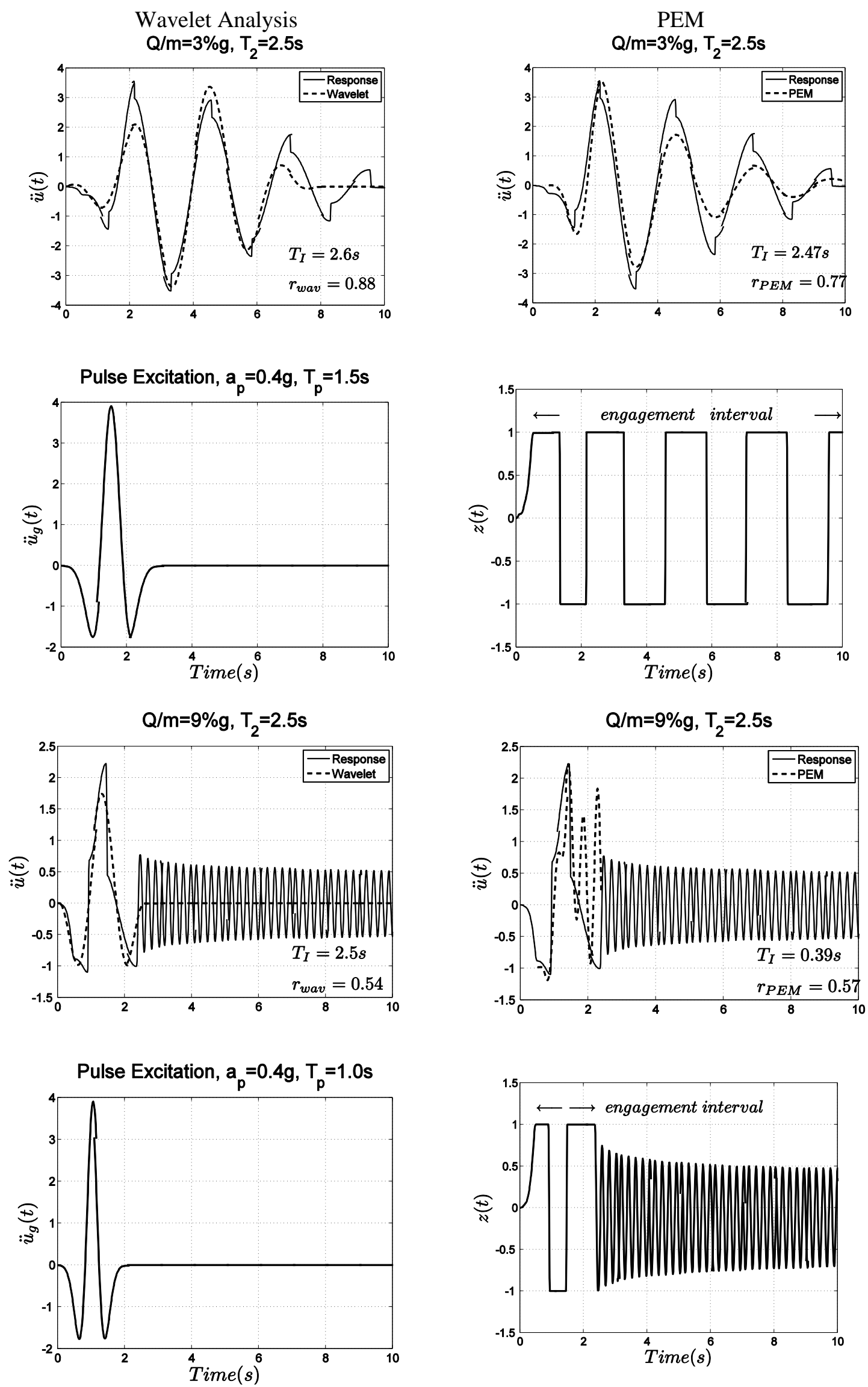

Figure 8. Matching the acceleration response histories above isolators with bilinear behavior with wavelet analysis (left) and the Prediction Error Method (right) which is applied only in the interval of the response history where the second slope, $K_{2}$ is engaged $(|z(t)|=1)$. 

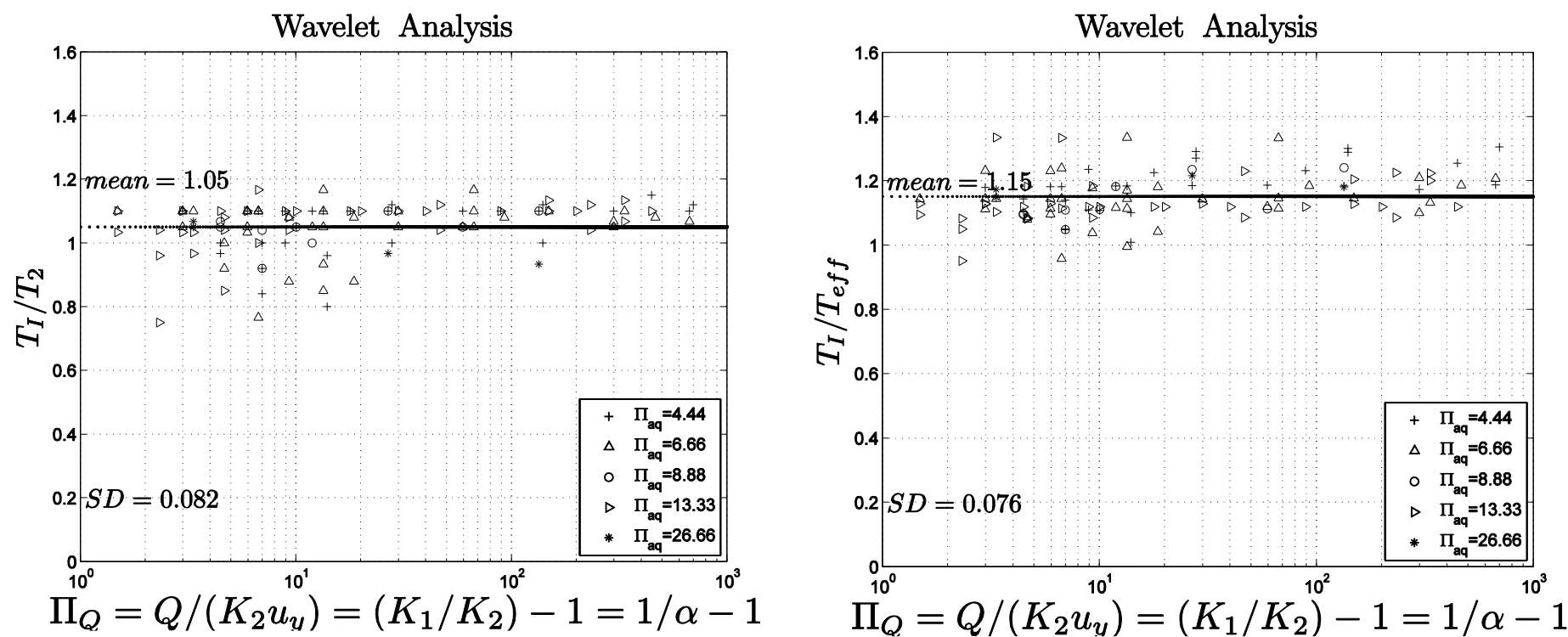

Figure 9. Values of the vibration period of bilinear isolation systems identified during forced vibration (symmetric Ricker pulses) with wavelet analysis.

Time Analysis-PEM

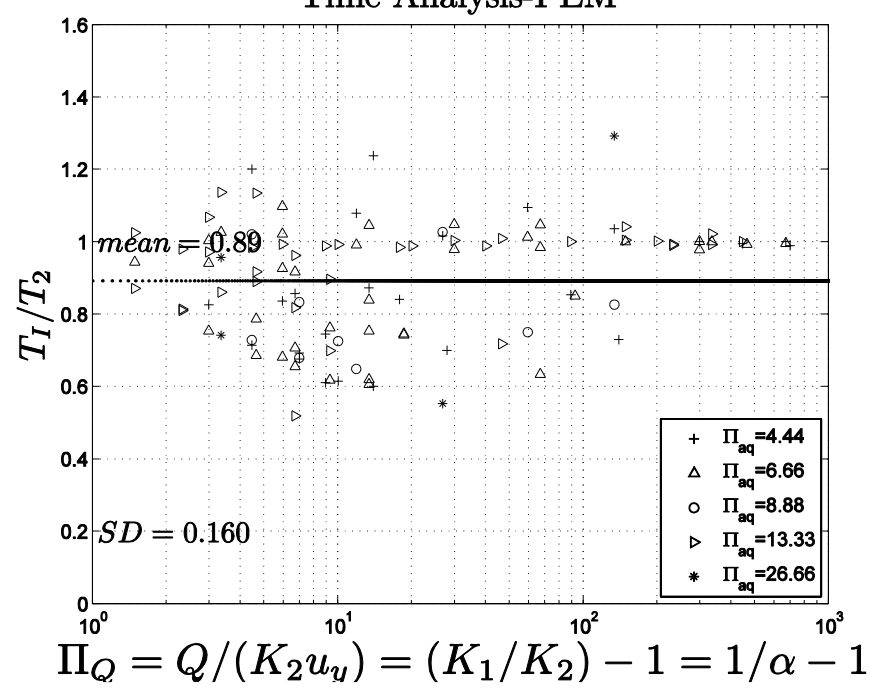

Time Analysis-PEM

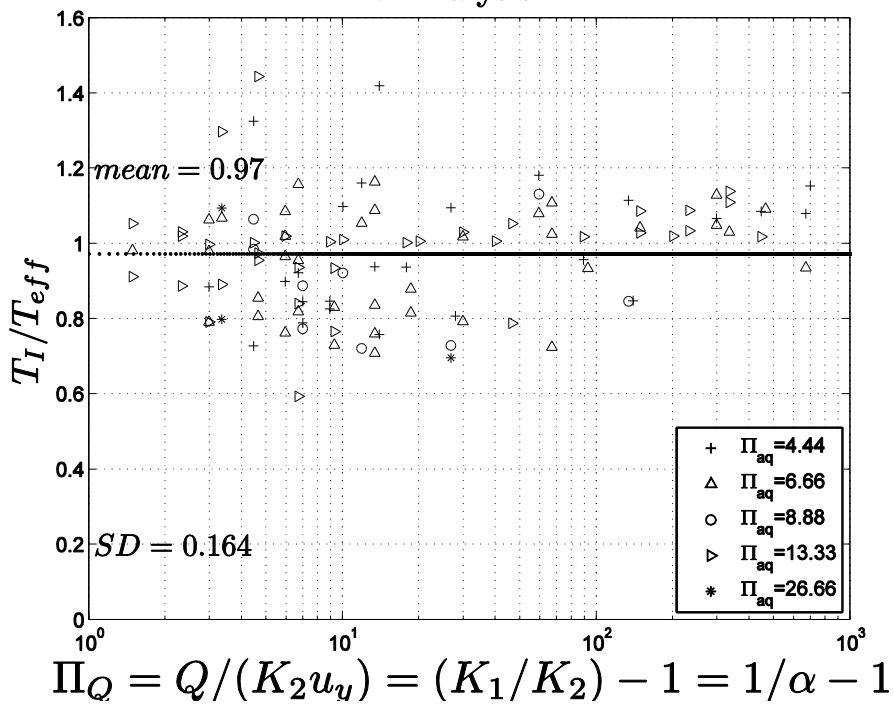

Figure 10. Values of the vibration period of bilinear isolation systems during forced vibration (symmetric Ricker pulses) extracted with the Prediction Error Method (PEM).

excitation. For the seismic response analysis we select six historic earthquake records listed in Table 2 which cover a wide range of spectral accelerations.

Under earthquake excitation the period that dominates the forced vibration response of the bilinear system - that is the isolation period, $T_{I}$, is a function of

$$
T_{I}=f\left(\frac{Q}{m}, u_{y}, T_{2} \text {, excitation parameters }\right)
$$

In analogy to the dimensional analysis presented for the case of forced vibrations under pulse excitation (see eq.33), equation (34) can be expressed in terms of dimensionless products

$$
\frac{T_{I}}{T_{2}}=\varphi\left(\frac{Q}{K_{2} u_{y}}, \text { dimensionless excitation parameters }\right)
$$

As in the case of pulse excitation, equation (35) indicates that the period which prevails during force vibration-that is the isolation period is equal to the period associated with the 

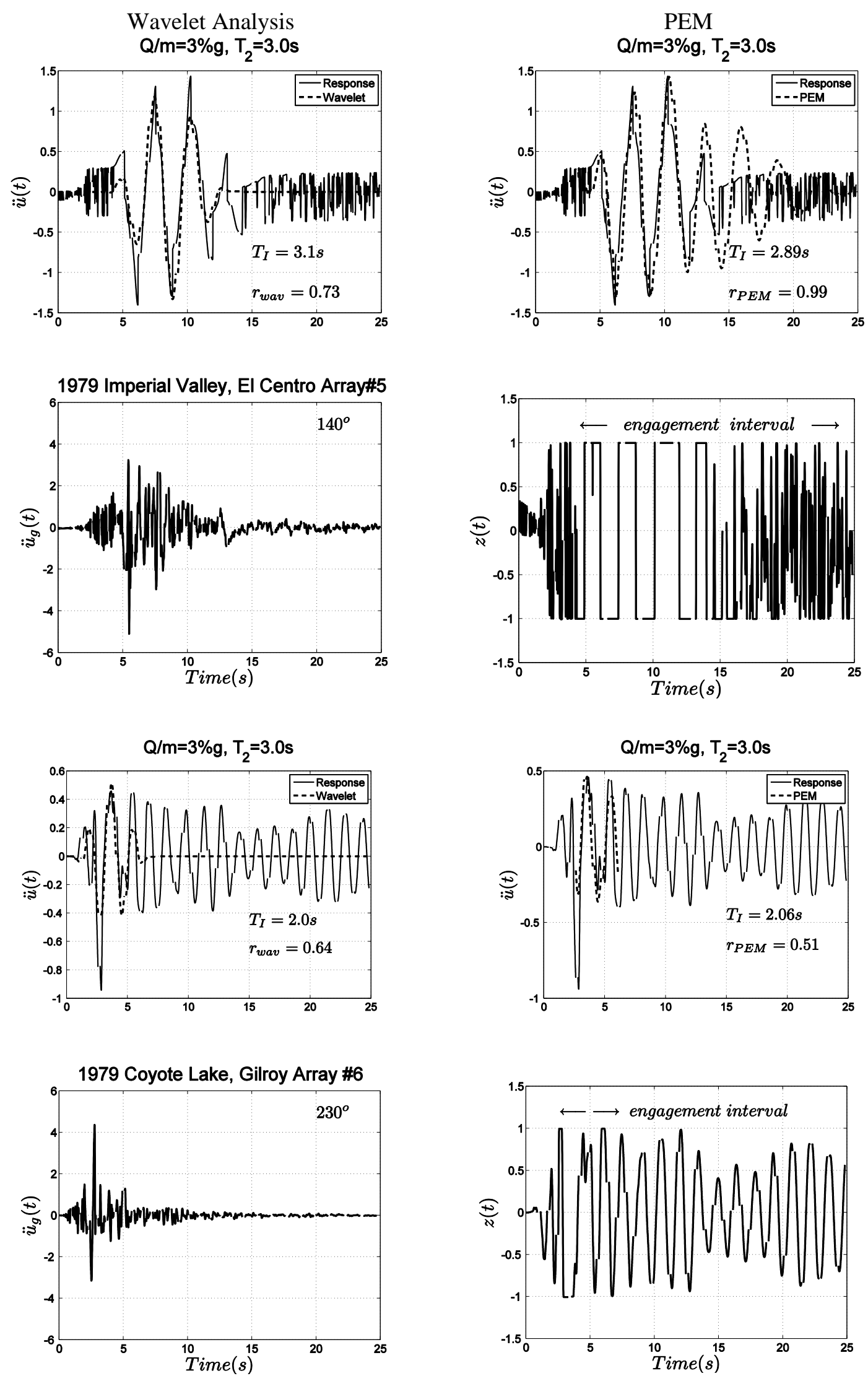

Figure 11. Matching the acceleration response histories above isolators with bilinear behavior with wavelet analysis (left) and the Prediction Error Method (right) which is applied only in the interval of the response history where the second slope, $K_{2}$ is engaged $(|z(t)|=1)$. 
second slope $T_{2}=2 \pi \sqrt{m / K_{2}}$, while being modified by some function $\varphi\left(\frac{Q}{K_{2} u_{y}}\right.$, dimensionless excitation parameters).

The identification of the "vibration" period of bilinear isolation systems subjected to earthquake excitation is first achieved with wavelet analysis.

Fig. 11 (left) plots with a dashed line the best matching Mavroeidis and Papageorgiou (M\&P) wavelet (Vassiliou and Makris 2011) on the acceleration response history of a bilinear system with strength $Q / \mathrm{m}=0.03 \mathrm{~g}$ and period that corresponds to the second slope $T_{2}=3 \mathrm{~s}$ when subjected to the El Centro Array\#5 ground motion recorded during the 1979 Imperial Valley earthquake. A number of bilinear systems have been selected to cover a wide range of the dimensionless product $\Pi_{Q}=Q / K_{2} u_{y}=1 / a-1$ and values of yield strength $Q /(m g)=3 \%, 5 \%, 7 \%$ and $9 \%$. Fig. 12(left) plots the computed isolation period $T_{I}$ normalized to the period associated with the second slope $T_{2}=2 \pi \sqrt{m / K_{2}}$ as a function if the dimensionless product $\Pi_{Q}=1 / a-1$ as extracted with wavelet analysis in which the daughter wavelet is the M\&P wavelet (Vassiliou and Makris 2011). Fig. 12 (left) shows that the mean value of $T_{I} / T_{2}=0.94$ is close to unity; nevertheless, the scattering of the data from the mean value is now appreciable yielding a value for the standard deviation, $S D=0.310$. Accordingly, there are several situations where the identification signals either from wavelet analysis or from the prediction error method (shown in Fig. 13) are incapable to yield a coherent vibration period. Fig. 12 (right) shows that when the computed isolation period, $T_{I}$, is normalized with the effective period, $T_{\text {eff }}$, the mean value is $T_{I} / T_{\text {eff }}=1.14$ while the scattering is even larger than in Fig. 12 (left), yielding a standard deviation $S D=0.360$.

Table 2. Earthquake records selected for this study.

Earthquake

1979 Coyote Lake, CA

1979 Imperial Valley, CA

1986 El Salvador

1992 Erzincan, Turkey

1992 Cape Mendocino, CA

1995 Aigion, Greece

\section{Record Station}

Gilroy Array \#6 230

El Centro Array \#5 140

Geot. Inv. Center 180

95 Erzincan

Cape Mendocino/000 OTE Building

\section{Magnitude, $M_{w}$}

5.7

6.5

5.5

6.9

7.2

6.2
$\operatorname{PGA}(\mathbf{g})$

0.43

0.52

0.48

0.52

1.49

0.54
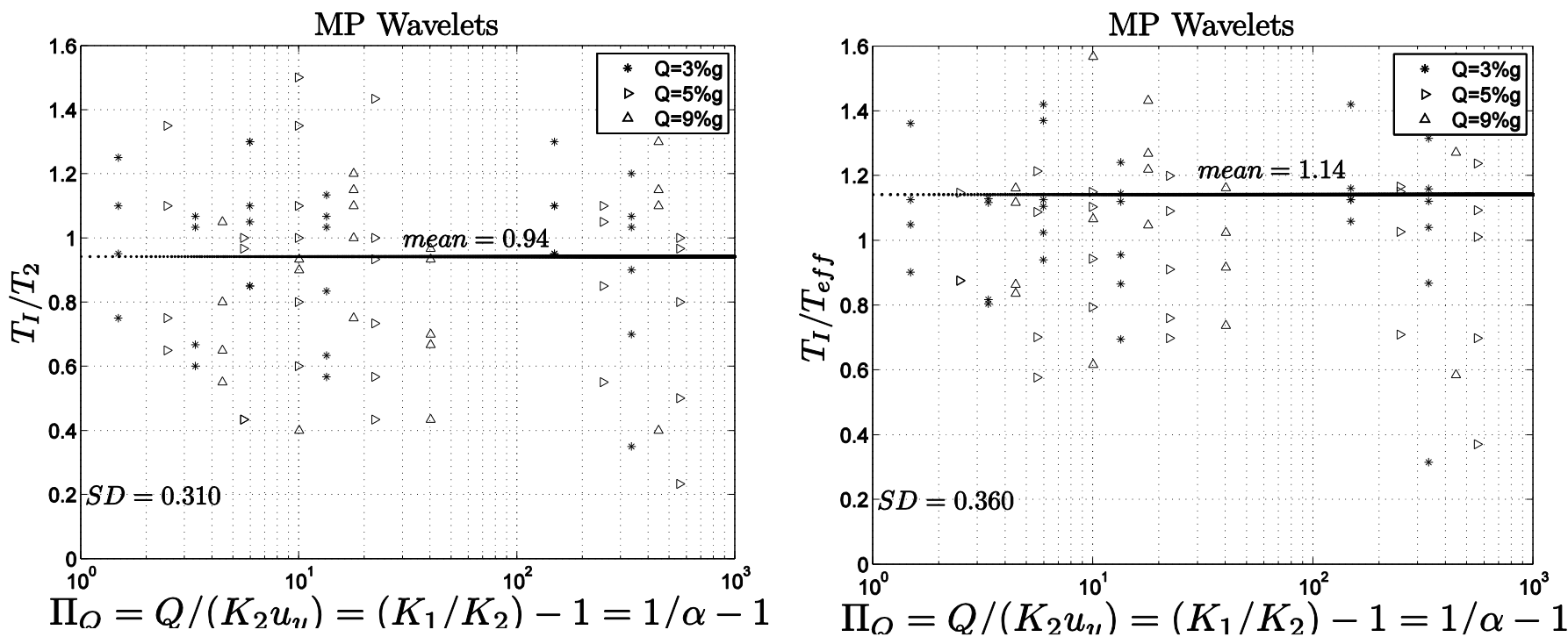

Figure 12. Values of the vibration period of bilinear isolation systems identified during forced vibration (recorded earthquake motions) with wavelet analysis. 

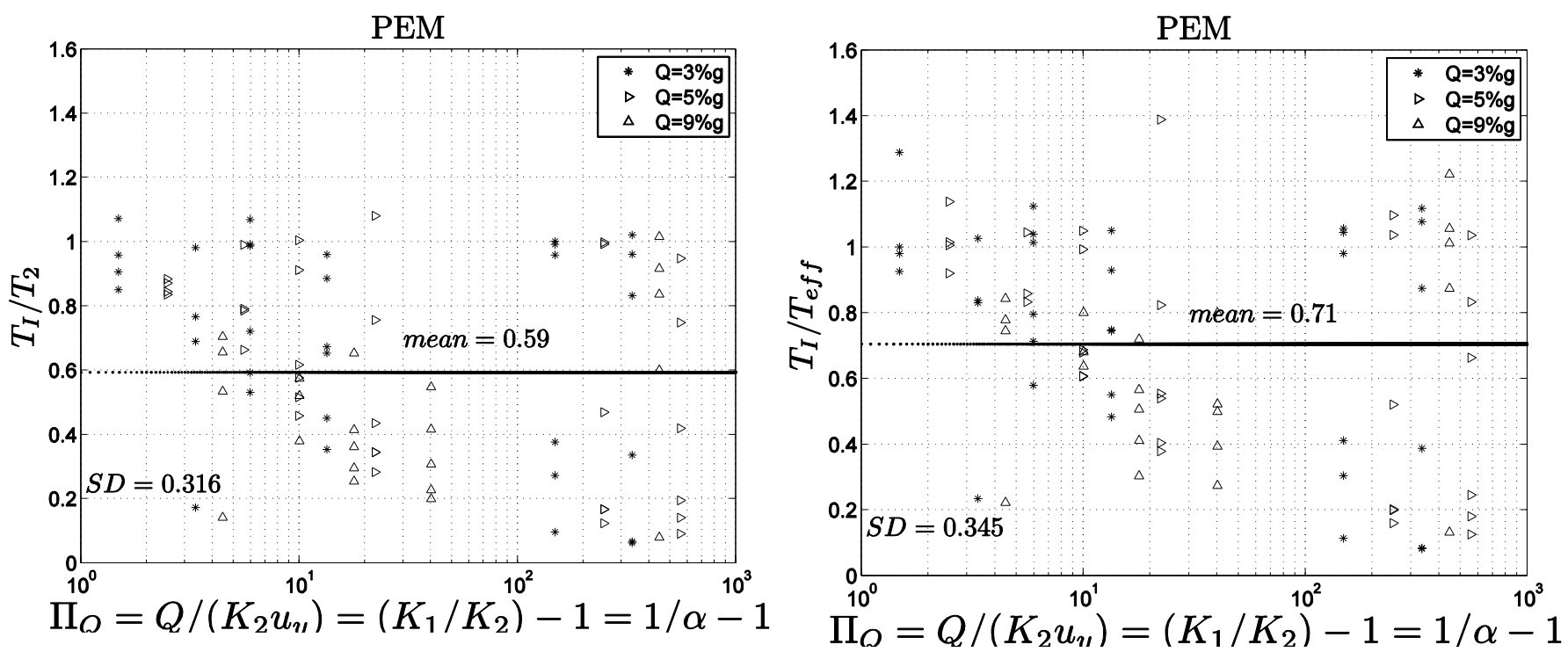

Figure 13. Values of the vibration period of bilinear isolation systems identified during forced vibration (recorded earthquake motions) with the Prediction Error Method (PEM).

\section{A Matching Index}

The forgoing analysis on the free vibration, forced vibration with a single-period mathematical pulses and forced vibration with recorded strong earthquake motions revealed that as the frequency content of the excitation widens and the intensity of the excitation fluctuates the standard deviations of the predicted "linear vibration period" of the bilinear system increases regardless whether this vibration period, $T_{I}$, is approximated with either the effective period, $T_{\text {eff }}=T_{2} \sqrt{1 /\left(1+Q /\left(K_{2} u_{\max }\right)\right)}$ or merely with the period associated with the second slope, $T_{2}$-which in general offers superior results. The wide scattering of some data points from the mean values of $T_{I} / T_{2}$ or $T_{I} / T_{\text {eff }}$ in Fig. 9, 10, 12 and 13 is because in the corresponding response histories the nonlinearities associated with the bilinear behavior dominate the response; therefore, the concept of associating a "vibration period" as required by the current design codes is meaningless. Interestingly, the scattering of data does not show any correlation with the normalized strength of the system. For instance, Fig. 11 (bottom-left) plots with dashed line the best matching M\&P wavelet on the acceleration response history of a bilinear system with strength $Q / m g=0.03$ and $T_{2}=3 s$ when subjected to the 1979 Coyote Lake, Gilroy Array \#5 record; while Fig. 11 (right) plots with a dashed line the response of the best matching equivalent linear system, as identified with PEM during the time interval that the second slope of the bilinear system is engaged. With the wavelet identification $T_{I}=2.0 \mathrm{~s}\left(T_{I} / T_{2}=0.66\right.$ and $\left.T_{I} / T_{\text {eff }}=0.81\right)$ while with the PEM identification $T_{I}=2.06 \mathrm{~s}\left(T_{I} / T_{2}=0.69\right.$ and $\left.T_{I} / T_{\text {eff }}=0.83\right)$. The ratios for $T_{I} / T_{2}$ or $T_{I} / T_{\text {eff }}$ given above in association with the assessment after visual observation of the scattering of all data in Fig. 12 and 13 suggest that the idea of associating a vibration period in several occasions should be abandoned. Consequently, we reach the conclusion that for bilinear isolation systems the "period of vibration" as expressed in most current design codes (AASHTO (1991), NZMWD (1983), FEMA (1998), Eurocode (2009) among others) can be identified only for certain combination of bilinear systems and ground motions. There is a class of response histories above isolators that are not capable to reveal any "vibration period". In this section we attempt to identify this class of response histories by proposing a matching index.

The idea behind developing a dependable matching index is that the wavelet signal or the PEM signal that is derived from the identification algorithms introduced earlier need to match 
to a reasonable extent the acceleration history of the bilinear system (accelerations above isolators). Accordingly, we introduce a matching index $r_{w a v}$ and a matching index $r_{P E M}$ as the ratio of the inner product of the best matching signal with the record normalized by the energy of the record,

$$
r_{\text {wav }}=\frac{\int_{0}^{T_{e}}\left|\psi(t) \ddot{u}_{b}(t)\right| d t}{\int_{0}^{T_{e}}\left[\ddot{u}_{b}(t)\right]^{2} d t}, 0<r_{\text {wav }}<1 \text { and } r_{P E M}=\frac{\int_{0}^{T_{e}}\left|a(t) \ddot{u}_{b}(t)\right| d t}{\int_{0}^{T_{e}}\left[\ddot{u}_{b}(t)\right]^{2} d t}, 0<r_{P E M}<1
$$

where $\psi(t)$ is the best matching wavelet, $a(t)$ is the acceleration history of the resulted PEM signal and $\ddot{u}_{b}(t)$ is the acceleration history of the bilinear system above isolators. Clearly,
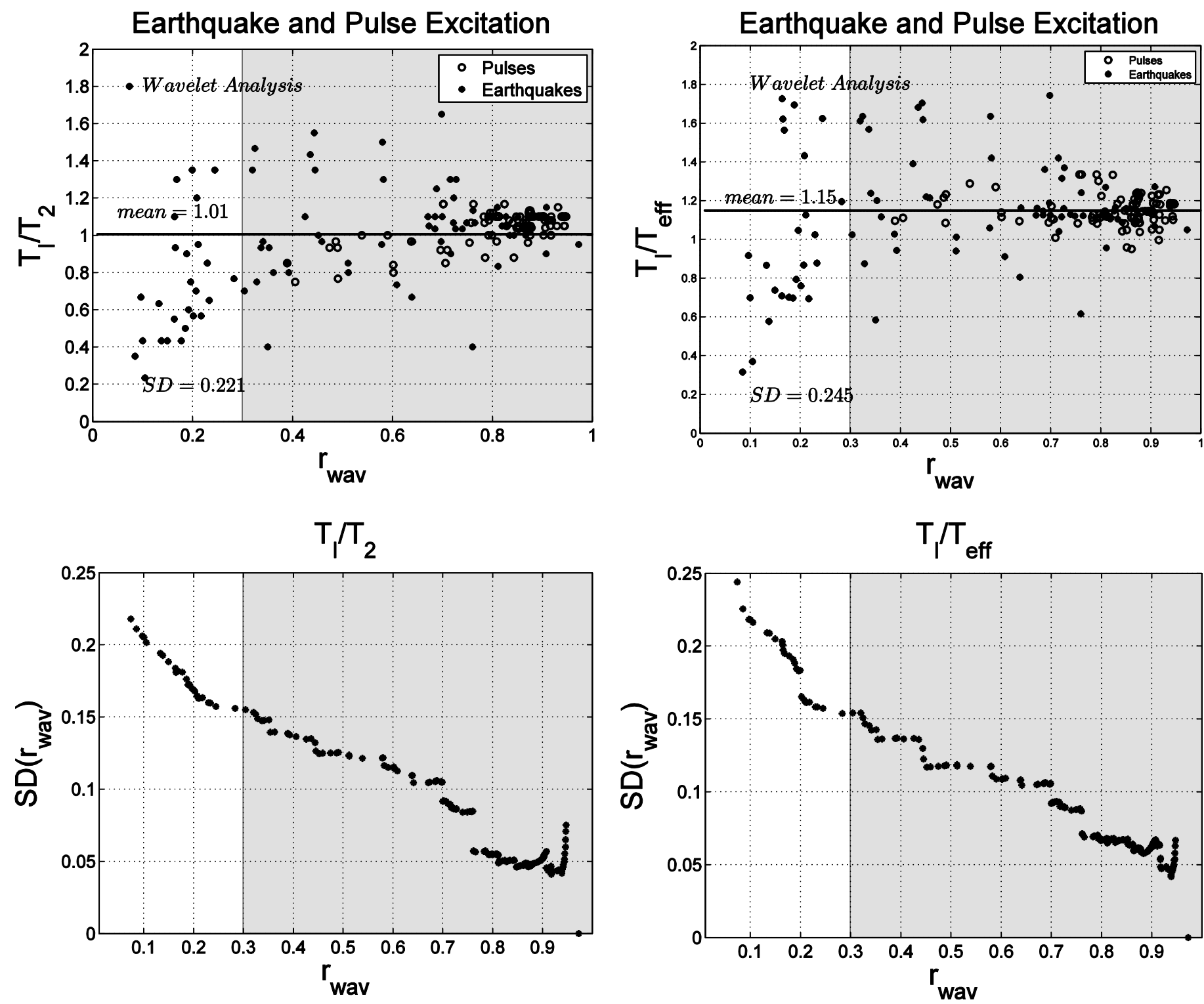

Figure 14. Top: Values of the vibration period of bilinear isolation systems identified during forced vibration (symmetric Ricker pulses and recorded ground motions) with wavelet analysis (all data shown in Figures 9 and 12) plotted vs the matching index, $r_{w a v}$. Bottom: Vales of the standard deviation, $S D\left(r_{\text {wav }}\right)$ of the data shown above from any given value of $r_{\text {wav }}$ up to $r_{\text {wav }}=1$. 
both $r_{w a v}$ and $r_{P E M}$ assume values between zero and one. Fig. 14 (top) plots the vibration periods of a wide range of bilinear systems identified during forced vibrations (pulse and earthquake excitations) with wavelet analysis (all data appearing in Fig. 9 and 12), where now they are plotted as a function of the matching index $r_{w a v}$ defined by equation (36). Fig. 14 (top) reveals that regardless if the data are normalized with $T_{2}$ or $T_{\text {eff }}$ their dispersion decreases as $r_{\text {wav }}$ increases. Fig. 14 (bottom) plots the standard deviation of the data shown in Fig. 14 (top) for any given value of $r_{w a v}$ up to $r_{w a v}=1$. For instance, the value of $S D \approx 0.15$ shown for $r_{w a v}=0.3$ is the standard deviation of all the data within $0.3 \leq r_{w a v} \leq 1$ (all points in the shaded region). Fig. 15 (top) plots the vibration periods of the bilinear systems identified during the forced vibration (pulse and earthquake excitation) with the prediction error method (all data appearing in Fig. 10 and 13), where now they are plotted as a function of the matching index $r_{P E M}$ defined by equation (36). Fig. 15 (bottom) plots the standard deviation of the data shown in Fig. 15 (top) from any given value of $r_{P E M}$ up to $r_{P E M}=1$. For instance, the value of $S D=0.15$ shown for $r_{P E M}=0.4$ is the standard deviation of all the data within the range $0.4 \leq r_{P E M} \leq 1.0$ (all points in the shaded region). Similar to Fig. 14, Fig. 15 reveals that regardless if the data are normalized with $T_{2}$ or $T_{\text {eff }}$ their dispersion decreases as $r_{P E M}$ increases.

Fig. 14 and 15 show that there are several response histories with $r_{w a v}$ or $r_{P E M}$-values as low as 0.3 or even less while, their corresponding "vibration period" departs from the mean value to such an extent that creates disproportional growth in the standard deviation when $r_{w a v}$ or $r_{P E M}$ approaches the axis origin.

Accordingly, there is a need to develop a procedure to separate the "good" response histories where the concept of associating a "vibration period" as required by the current design codes is meaningful. This separation is most useful in system identification studies which attempt to extract the isolation period of seismically isolated bridges from recorded signals above and below isolators.

\section{Selection of the "Good" Response Histories}

The final goal of this paper is to separate the response histories of bilinear systems where the concept of associating a "vibration period" as required by the current design codes is meaningful; from the response histories where the concept of associating a vibration period is meaningless. This separation can be achieved if one observes the plots of the standard deviations, $S D\left(r_{\text {wav }}\right)$ and $S D\left(r_{P E M}\right)$, shown in Fig. 14(bottom) and 15(bottom) which exhibit a growing values of $S D$ when $r_{w a v}<0.3$ or when $r_{P E M}<0.4$. Our aim is to take the best elements offered by the wavelet analysis and by the Prediction Error Method (PEM). We return now to Fig. 11 (bottom) where the isolation system with $Q / m g=0.03$ and $T_{2}=3 \mathrm{~s}$ when excited by the 1979 Coyote Lake, Gilroy Array\#6 record experienced few and small displacements beyond the yield displacement $u_{y}$ (see the few and small plateaus in the zhistory). In such situations the response history above isolators contains poor information regarding the isolation period, $T_{2}$, associated with the second slope of the bilinear system. Accordingly, when evaluating a response history we need also to include a measure that indicates to what extent the second slope of the bilinear system 

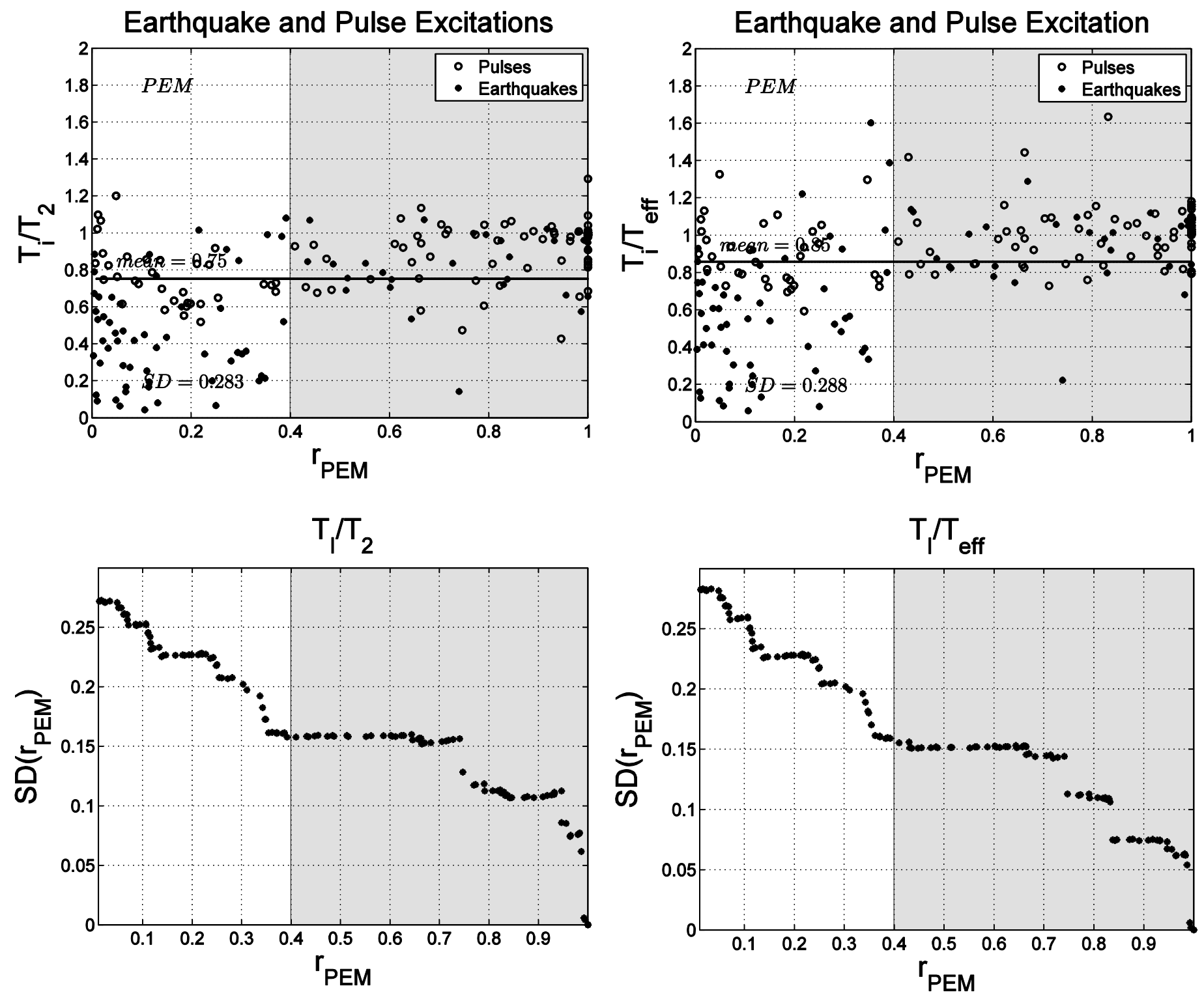

Figure 15. Top: Values of the vibration period of bilinear isolation systems identified during forced vibration (symmetric Ricker pulses and recorded ground motions) with PEM (all data shown in Figures 10 and 13) plotted $v$ s the matching index, $r_{P E M}$. Bottom: Vales of the standard deviation, $S D\left(r_{P E M}\right)$ of the data shown above from any given value of $r_{P E M}$ up to

$$
r_{P E M}=1 \text {. }
$$

was engaged. This need is served with the engagement ratio $\sum N_{i}$ plateau/ $\sum N_{i}$ where $N_{i}$ plateau are the number of data points within the segment where $|z(t)|=1$ and $N_{i}$ are all the data points between the first and last yielding of the system during the excitation.

Our selection process proceed by taking only the data in Fig. 14 with $r_{w a v}>0.3$ or the data in Fig. 15 with $r_{P E M}>0.4$ and re-evaluate them by computing an improved matching index which incorporates the engagement ratio

$$
r=\frac{1}{\sqrt{2}} \frac{\sum N_{i, \text { plateau }}}{\sum N_{i}} \sqrt{{r_{\text {wav }}{ }^{2}+r_{P E M}{ }^{2}}^{2}}, \quad 0 \leq r \leq 1
$$


Any response history with either $r_{w a v}>0.3$ or $r_{P E M}>0.4$ is retained as a potentially "good" response history and its identified vibration period appears in Fig. 16.

Accordingly, Fig. 16 (top) shows the vibration periods extracted from the responses of bilinear systems that passed the first screening ( $r_{\text {wav }}>0.3$ or $\left.r_{P E M}>0.4\right)$ and are plotted as a function of the improved matching index, $r$, given by equation (37). Therefore, the number of data shown in Fig. 16 is smaller than the number of the data appearing in Fig. 14 or Fig. 15. It is interesting to note in Fig. 16, several response histories which passed the first screening ( $r_{w a v}>0.3$ or $\left.r_{P E M}>0.4\right)$ their improved $r$ index is now poor $(r<0.3)$-therefore, the need for a more refine selection. Fig. 16 (bottom) plots the standard deviation of the data shown in Fig. 16 (top) from any given value of $r$ up to $r=1$. For instance, the value of $S D=0.1$ shown when $r=0.5$ is the standard deviation of all the data within $0.5 \leq r \leq 1.0$.
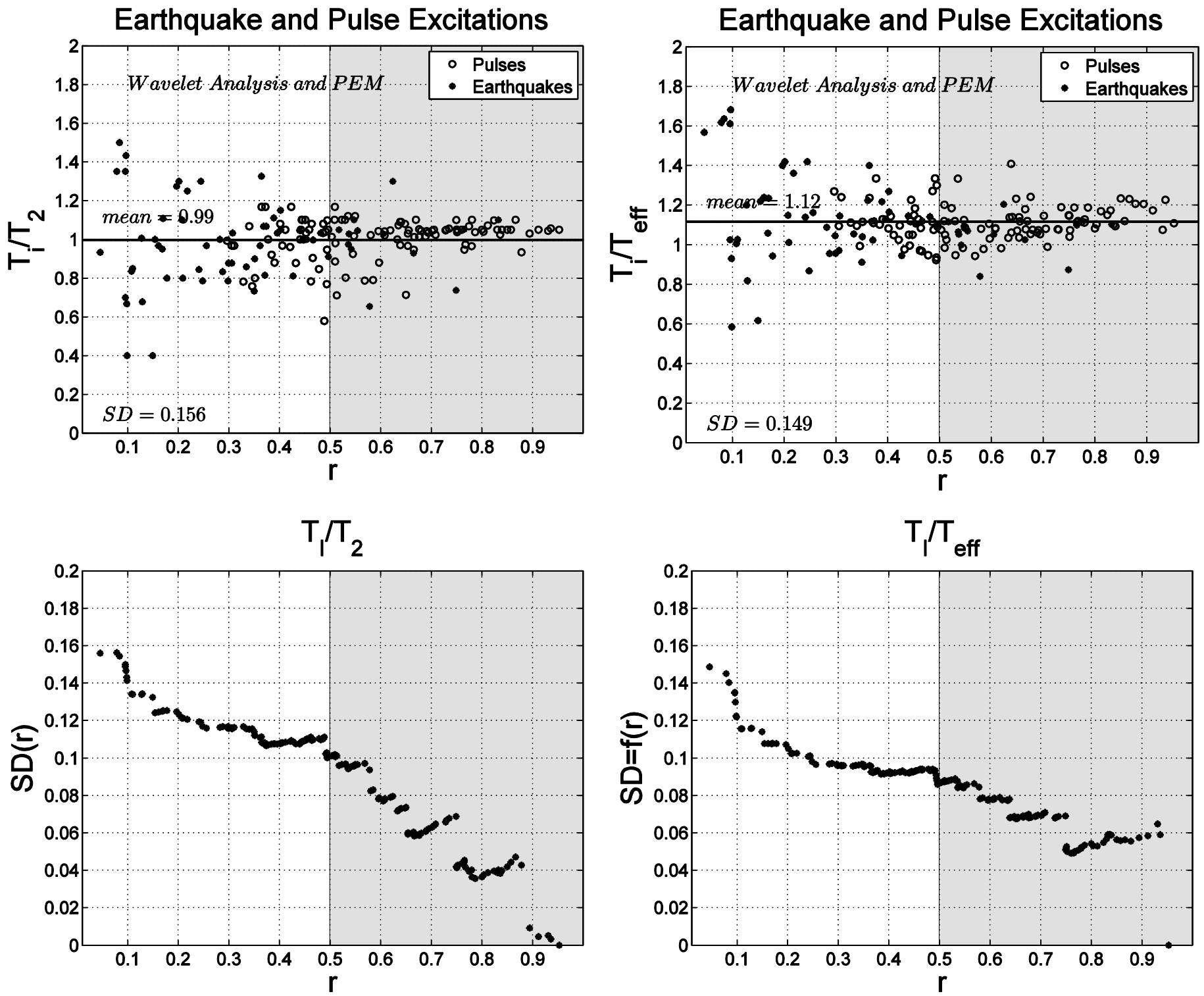

Figure 16. Top: Values of the vibration period of bilinear isolation systems under forced vibrations with matching index $r_{\text {wav }}>0.3$ or $r_{P E M}>0.4$ plotted $v$ s the improved index $r$ given by equation (36). Bottom: Values of the standard deviation $S D(r)$ of the data shown above from any given value of $r$ up to $r=1$. 
Interestingly, for values $r<0.5$ the values of the standard deviation $S D(r)$ exhibits a finite jump above the value of $S D=0.1$ and for values of $r<0.35, S D(r)$ exhibits a rapid increase as we move towards the axis origin.

In conclusion, recorded time histories with $r>0.5$ are characterized as "good" response histories and the identified period $T_{I}$ is strongly correlated with the period associated with the second slope of the bilinear system, $T_{2}$ or with the effective period $T_{\text {eff }}$. In contrast, this analysis shows that for recorded time histories above isolators with $r<0.5$, the concept of assigning a "vibration period" becomes feeble and shall be abandoned.

\section{Conclusions}

This paper examines whether the "effective period" of bilinear isolation systems, as defined invariably in most current design codes, expresses in reality the period of vibration that appears in the horizontal axis of the response spectrum. The study employs Fourier and Wavelet analysis together with a time domain identification method for linear systems known as the Prediction Error Method (PEM) to process the vibration response of a wide collection of bilinear isolation systems subjected to pulse and earthquake excitations.

When the response history of the bilinear system exhibits a coherent oscillatory trace with a narrow frequency band as in the case of free vibration of forced vibrations from most pulselike excitations, the paper shows that the "effective period" $=T_{\text {eff }}$ of the bilinear isolation system is a dependable estimate of its vibration period. At the same time the paper concludes that the period associated with the second slope of the bilinear system $=T_{2}$ is an even better approximation of the "vibration period" regardless of the value of the dimensionless strength $Q /\left(K_{2} u_{y}\right)=1 / \alpha-1$ of the system. Consequently, this study concludes that whenever the concept of associating a "vibration period" is meaningful the "effective" period, $T_{\text {eff }}$ can be replaced with $T_{2}$ which is a period that is known a priori (no iterations are needed) and offers in general superior results. This finding serves both simplicity and a more rational estimation of maximum displacement. Simplicity is served because instead of looking for $T_{\text {eff }}-\mathrm{a}$ quantity that derives from the non-existing $K_{\text {eff }}$, for which iterations are needed to be approximated, the paper shows that the period associated with the second slope of the bilinear system $=T_{2}$ (that is known a priori-no iterations are needed) is a better single-value descriptor of the frequency content of the dynamic response of a bi-linear isolation system. Given that $T_{2}$ is always longer than $T_{\text {eff }}$ the peak inelastic displacement does no run the risk to be underestimated.

Most importantly, the paper shows that as the frequency content of the excitation widens and the intensity of the acceleration response history fluctuates more randomly the computed vibration period of the bilinear isolation system exhibits appreciable scattering from the computed mean value. This scattering of the identified period values is due to the nonlinear nature of the response signal; and therefore, for this class of response histories the expectation of the design codes to identify a "linear vibration period" has marginal engineering merit.

The paper develops a physically motivated matching index that permits the separation of the response histories of bilinear systems where the concept of associating a "vibration period" is meaningful from those where the concept of associating a "vibration period" is feeble.

In conclusion, the engineering merit of the effective period $T_{\text {eff }}$ of bilinear isolation systems as given by equation (5) is marginal given that: (a) whenever the concept of associating a "vibration period" is meaningful $(r>0.5)$, this "vibration period" can be approximated in a superior way with the period associated with the second slope $=T_{2}$ as (see Fig. 5,7 and 9); and (b) when the matching index $r$ is low (say $r<0.5$ ) the concept of associating a "vibration period" has marginal engineering value. 


\section{Acknowledgements}

Partial financial support has been provided by the EU research project "DARE" ("SoilFoundation-Structure Systems Beyond Conventional Seismic Failure Thresholds: Application to New or Existing Structure and Monuments"), which is -Advanced Grant, under contract number ERC-2---9-AdG228254-DARE to Prof. G. Gazetas.

\section{References}

1. Addison P. S. (2002) The illustrated wavelet transform handbook, Institute of Physics Handbook.

2. Anti-seismic devices (2009), European Standard, FprEN 15129, Eurocode.

3. Aström, K. J., and Bohlin T. (1965) "Numerical identification of linear dynamic systems from normal operating records". IFAC Symposium on Self-Adaptive Systems, Teddington, England.

4. Buckle I.G., and Mayes R.L., (1990) "Seismic Isolation: History, application and performance -A world view". Earthquake Spectra Journal, 6(2).

5. Caughey, T.K., (1960) "Random excitation of a system with bilinear hysteresis". Journal of Applied Mechanics, 27:649-652.

6. Caughey, T.K., (1963) "Equivalent linearization techniques". Journal of Acoustical Society of America, 35:1706-1711.

7. Constantinou M.C., Mokha, A.S., Reinhorn, A.M., (1990) "Teflon bearing in base isolation. II: modeling". Journal of Structural Engineering (ASCE), 116:455-474.

8. Crandall, S.H. (2006) "A half-century of stochastic equivalent linearization". Journal of Structural Control and Health Monitoring, 13:27-40.

9. Design of lead-rubber bearings (1983) Civil division publication 818/A, New Zealand Ministry of Works and Development, Wellington, New Zealand.

10. FEMA 310 (1998), Handbook for the Seismic Evaluation of Buildings - A Prestandard, ASCE.

11. Garini, E., Gazetas, G., and Anastasopoulos, I. (2010) "Accumulated Assymetric Slip Caused by Motions Containing Severe 'Directivity' and 'Fling' Pulses". Geotechnique, 70, in press.

12. Guide specifications for seismic isolation design (1991) American Association of State Highway and Transportation officials, Washington, D.C.

13. Hwang J.S., and Chiou, J.M., (1996) "An equivalent linear model of lead-rubber seismic isolation bearings". Journal of Engineering Structures, 18(7): 528-536.

14. Hwang, J.S., and Sheng L.H. (1993) "Equivalent elastic seismic analysis of baseisolated bridges with lead-rubber bearings". Journal of Engineering Structures, 16(3):201-209.

15. Hwang, J.S., and Sheng L.H. (1994) "Effective stiffness and Equivalent Damping of Base-Isolated Bridges". Journal of Structural Engineering, 119(10):3094-3101.

16. International Code Council (2000), International Building Code.

17. Iwan W.D., and Gates N.C., (1979) "The effective period and damping of a class of hysteretic structures". Journal of Earthquake Engineering and Structural Dynamics, 7:199-211.

18. Iwan W.D., (1980) "Estimating inelastic response spectra from response spectra". Journal of Earthquake Engineering and Structural Dynamics, 8:375-388.

19. Kelly, J.M., Eidinger J.M.,and Derham C.J., (1977) A practical soft story system. Report No. UCB/EERC-77|27, Earthquake Engineering Research Center, University of California, Berkeley, California.

20. Kelly, J.M., (1986) "Seismic base isolation: Review and bibliography". Journal of Soil Dynamics and Earthquake Engineering, 5: 202-216.

21. Ljung L. (1987) System Identification-Theory for the User, Prentice-Hall, New Jersey. 
22. Ljung L. (1994) "State of the Art in Linear System Identification: Time and Frequency Domain Methods". Proceedings of '04 American Control Conference, 1:650-660.

23. Ljung L. (2002) "Prediction Error Estimation Methods". Circuits Systems Signal Processing, 21: 11-21.

24. Makris, N., and Black, C. J. (2004a) "Dimensional analysis of rigid-plastic and elastoplastic structures under pulse-type excitations". J.Eng. Mech., 130: 1006-1018.

25. Makris, N., and Black, C. J. (2004b) "Dimensional analysis of bilinear oscillators under pulse-type excitations". J. Eng. Mech., 130: 1019-1031.

26. Makris N., and C. Black (2004c) "Evaluation of peak ground velocity as a "good" intensity measure for near-source ground motions". J. Eng. Mech., ASCE 130: 10321044.

27. Makris N, Chang S. (2000) "Effect of viscous, viscoplastic and friction damping on the response of seismic isolated structures". Journal of Earthquake Engineering and Structural Dynamics, 29(1): 85-107.

28. Makris N., and Vassiliou M. F., (2011) “The existence of 'complete. similarities' in the response of seismic isolated structures subjected to pulse-like ground motions and their implications in analysis". Journal of Earthquake Engineering and Structural Dynamics, 40(10):1103-1121.

29. Mallat S. G. (1999) A wavelet tour of signal processing. Academic Press.

30. MATLAB. High-performance Language Software for Technical Computation. The MathWorks, Inc: Natick, MA, 2002.

31. Mayes R.L., Buckle, I.G., Kelly, T.E., and Jones, L.R. (1991) "AASHTO Seismic Isolation Design Requirements for Highway Bridges". Journal of Structural Engineering, 118(1):284-304.

32. Mokha, A.S., Constantinou, M.C., Reinhorn, A.M., (1990) "Teflon bearing in base isolation. I: testing". Journal of Structural Engineering (ASCE), 116:438-454.

33. Naeim, F., and Kelly, J.M., (1999). Design of Seismic Isolated Structures. New York: Wiley Publications.

34. Naeim, F (2001) The seismic design handbook, Springer.

35. Ricker, N. (1943) "Further developments in the wavelet theory of seismogram structure". Bull. Seism. Soc. Am., 33:197-228.

36. Ricker N (1944) "Wavelet functions and their polynomials". Geophysics 9:314-323.

37. Roberts J.B and Spanos P.D. (2003) Random vibration and statistical Linearization. New York: Dover Publications.

38. Vassiliou, M., F., and Makris, N., (2011) "Estimating Time Scales and Length Scales in Pulselike Earthquake Acceleration Records with Wavelet Analysis". Bull. Seism. Soc. Am., 101(2):596-618.

39. Veletsos A.S., and Newmark, N.M., (1960) "Effect of inelastic behavior on the response of simple systems to earthquake motions". In: Proceedings of the second world conference on earthquake engineering, Japan, 2:895-912.

40. Veletsos A.S., Newmark N.M., Chelepati C.V. (1969) "Deformation spectra for elastic and elastoplastic systems subjected to ground shock and earthquake motions". Proceedings of the $3^{\text {rd }}$ World Conference on Earthquake Engineering, vol. II, Wellington, New Zealand: 663-682.

41. Veletsos A.S., Vann W.P. (1971) "Response of ground-excited elastoplastic systems". Journal of Structural Division (ASCE), 97(ST4):1257-1281.

42. Wen Y., K. (1975) “Approximate method for nonlinear random vibration”. Journal of Engineering Mechanics, 101(4):389-401.

43. Wen Y., K. (1976) "Method for random vibration of hysteretic systems". Journal of Engineering Mechanics, 102(2):249-263. 